\title{
A Mountain Pass for Reacting Molecules ${ }^{1}$
}

\author{
Mathieu LEWIN \\ CEREMADE, CNRS UMR 7534, Université Paris IX Dauphine, Place du \\ Maréchal de Lattre de Tassigny, 75775 Paris Cedex 16, France \\ E-mail: lewin@ceremade.dauphine.fr
}

\begin{abstract}
In this paper, we consider a neutral molecule that possesses two distinct stable positions for its nuclei, and look for a mountain pass point between the two minima in the non-relativistic Schrödinger framework.

We first prove some properties concerning the spectrum and the eigenstates of a molecule that splits into pieces, a behaviour which is observed when the Palais-Smale sequences obtained by the mountain pass method are not compact. This enables us to identify precisely the possible values of the mountain pass energy and the associated "critical points at infinity" (a concept introduced by Bahri [2]) in this non-compact case.

We then restrict our study to a simplified (but still relevant) model: a molecule made of two interacting parts, the geometry of each part being frozen. We show that this lack of compactness is impossible under some natural assumptions about the configurations "at infinity", proving the existence of the mountain pass in these cases. More precisely, we suppose either that the molecules at infinity are charged, or that they are neutral but with dipoles at their ground state.
\end{abstract}

AMS Subject Classification: 35B38, 35Q40, 35J10, 49J35, 81V70, 81V55, 81Q05, 49S05.

Keywords: mountain pass, critical points, critical points at infinity, Morse index, quantum mechanics, ground state, excited state, quantum chemistry, reaction path, transition state.

\footnotetext{
${ }^{1}$ Published in Ann. Henri Poincaré 5 (2004), 477-521.
} 


\section{Introduction}

In this paper, we study in the non-relativistic quantum Schrödinger framework the case of a molecule that possesses two distinct stable positions for its nuclei, as this is for instance the case for $\mathrm{HCN}$ and $\mathrm{CNH}$. Our purpose is somewhat simple: can we obtain a critical point of the energy by using the classical mountain pass method between the two minima? Experiment suggests that this is the case (at least for the $\mathrm{HCN} \leftrightarrow \mathrm{CNH}$ reaction). Indeed, such mountain pass points are frequently computed by chemists who need to understand the possible behaviour of the molecule: it corresponds to a "transition state" during an infinitely slow reaction leading from one minimum to the other. But as far as we know, this problem has never been tackled from the mathematical point of view for the $N$-body quantum problem, or even in the context of the classical Hartree or Thomas-Fermi type models which are approximations of the exact theory.

For a neutral molecule, the proof that there is a minimum with regards to the position of the nuclei can be found in the fundamental work of E.H. Lieb and W. E. Thirring [25] for the Schrödinger model, and in a series of papers by I. Catto and P.-L. Lions [4, 5, 6, 7] for approximate models (Hartree or Thomas-Fermi type), the latter being really more complicated due to the non linearity of these models. In these two works, the authors had to prove that minimizing sequences are compact, the non-compactness behaviour being related to the fact that the molecule can split into parts, each moving away from the others. Remark that binding does not occur for the Thomas-Fermi model (see the works of E. Teller [31], E.H. Lieb and B. Simon [24], and the references in [4]) and that the result is not known for the Hartree-Fock model, except in very special cases [7].

Let us make some comment on a tool used in these proofs that cannot be simply adapted to our setting. A common idea in these two works is to average over all the possible orientations of each piece in order to simplify the computation of the interaction energy between them, by suppressing the multipoles. To show that the energy can be lower than the energy at infinity, a new term using the correlation between the electrons is then created in [25] to obtain a Van Der Waals term of the form $-C / R^{6}(R$ is the distance between the molecules), while a very detailed computation of the (exponentially small) combined energy is done in $[4,5,6,7]$ to conclude that the system can bind. Because of the preliminary averaging, the conclusion is that there exists some orientation of the molecules for which this is true, but this position is unknown a priori.

In the case of the mountain pass method that we propose here, the noncompactness is obviously also due to a possible splitting of the molecule. However, we want to insist on the fact that we cannot use in this setting the same idea of averaging over all the rotations of the molecules, because we have to pull down the energy along a path. In other words, a comparison between the energies is not sufficient to conclude, and a precise information on the directions on which the energy decreases is needed. 
This is why we failed to treat the problem in its full generality and we had to add some hypothesis about the configurations "at infinity". Nevertheless, we wish to ameliorate this first work in the future, and hope that it will stimulate further results.

The results proved in this paper are the following.

First, we study the spectrum and the eigenfunctions of the Hamiltonian when a molecule splits into parts. We obtain some bounds on the eigenvalues and the bottom of the essential spectrum which allow to show that the "electrons remain in the vicinity of the nuclei" when a fixed excited state is studied. In other words, no electron is lost during the process. This is obtained by a non-isotropic exponential decay of the electronic density, which is shown to be uniform when the distance between the molecules grows. We also specify the behaviour of the associated wavefunctions and define the "critical points at infinity", a concept introduced by A. Bahri [2]. Some parts of this first result are necessary for our min-max problem.

Then, we prove a result that enables to identify the possible behaviour of the non-compact min-maxing paths. As it is suggested by the intuition, it is shown that the optimal energy of the mountain pass corresponds in this case to a system where the molecule is split into independent parts (the electrons are shared among them), each being at its ground state. This Morse information on the critical points at infinity is rather intuitive.

As announced, we were unable to treat the general case and we end this article by showing that this non-compactness behaviour is impossible in the special case of two interacting molecules with fixed nuclei. This is done under the hypothesis that we are in the easy case of two charged molecules at infinity, or in the more difficult case of two neutral molecules at infinity, but with dipoles at their ground state. This enables to obtain the required result for many practical situations. As explained before, the crucial step is to evaluate the interaction energy between the molecules and we use here a multipole expansion, even for wavefunctions that are not a simple tensor product of two ground states as in [25]. Finally, the expected result is deduced from the fact that the critical points of the dipole/dipole interaction energy which have a nonnegative energy have a Morse index which is at least 2.

From a practical point af view, the study of this mountain pass method is really important. As mentioned above, the main idea is that a path leading from one minimum to the other represents an infinitely slow chemical reaction. The mountain pass energy is then interpreted as the lowest energy threshold for the reaction to happen. The numerical computation of this energy and of the optimum (even the whole path) is then a prime necessity for chemists, who have to understand the possible behaviours of the molecule (see for instance $[28,9]$ for chemical and numerical aspects).

However, chemists only consider paths on which the molecule is at its ground state all along it, which leads to obvious problems of smoothness in the case of degeneracy of the first eigenvalue of the Hamiltonian, and can obstruct convergence. For mathematical reasons, we were thus forced to abandon this hy- 
pothesis and relax the problem by considering that the wavefunction can vary independently of the nuclear geometry, in order to obtain a critical point with respect to nuclei's variations. Since we shall show that our min-max energy is in fact the same that the one used in practice, this approach could also be interesting for numerical computations.

We conclude with a few words on the mathematical tools used in this paper. As in $[4,5,6,7]$, the proof is guided by P.-L. Lions' Concentration-Compactness ideas [26], although the localization of the electrons is given by the uniform exponential decay of Theorem 2, and not by this theory. Let us remark that the physical intuition is somewhat often related to the behaviour of the electronic density. For instance, when the molecule splits into parts, the latter becomes a sum of functions localized near the nuclei. But this point of view is not sufficient to understand the problem since the main object is not the density, but the wavefunction. The latter will not split into sums, but into sums of tensor products of wavefunctions in lower dimensions (see the work of G. Friesecke [10] for a very clear explanation of this phenomenon). Therefore, we use a variant of $N$-body geometric methods for Schrödinger operators [30, 29, 18] that enables to relate the behaviour of the wavefunction to those of the associated electronic density. This method is used in [10] and enabled G. Friesecke to notice an interesting link between the celebrated HVZ Theorem $[18,32,33]$ and the Concentration-Compactness method [26].

Moreover, the HVZ Theorem (which enables to identify the bottom of the spectrum as the ground state energy of the same system but with an electron removed), and Zhislin's Theorem (which states the existence of excited states for positive or neutral molecules) are abundantly used in this article.

Finally, we use the results and methods developed by G. Fang and N. Ghoussoub $[8,14]$ which enable to obtain Morse information on the Palais-Smale sequences, related to the fact that the deformed object are paths (i.e. deformations of $[0 ; 1])$. We also use the duality theory developed in $[14]$ which permits to locate critical points.

The paper is organized as follows. In the next section, we describe the model in detail and recall known results on the Hamiltonian and its eigenfunctions. Then, in section 2, we present our results without proof: for the sake of clarity, we have brought all the proofs together in the last section.

Acknowledgments: I would like to thank Éric Séré for his constant attention and helpful remarks.

\section{The model}

\subsection{Framework}

We consider here a positive or neutral molecule with $N$ non relativistic electrons, and $M$ nuclei of charges $Z_{1}+\cdots+Z_{M} \geq N$. The nuclei are supposed to be correctly described by a classical model (Born-Oppenheimer approximation) 
and are thus represented as pointwise charges at $R_{1}, \ldots, R_{M} \in \mathbb{R}^{3}$. In what follows, we let

$$
R=\left(R_{1}, \ldots, R_{M}\right) \in \Omega:=\left(\mathbb{R}^{3}\right)^{M} \backslash\left(\cup_{i \neq j}\left\{R_{i}=R_{j}\right\}\right)
$$

and

$$
Z=\left(Z_{1}, \ldots, Z_{M}\right) \in\left(\mathbb{N}^{*}\right)^{M}, \quad|Z|=Z_{1}+\cdots+Z_{M} \geq N .
$$

The system is described by the purely coulombic $N$-body Hamiltonian

$$
\begin{gathered}
H^{N}(R, Z)=\sum_{i=1}^{N}\left(-\frac{1}{2} \Delta_{x_{i}}+V_{R}\left(x_{i}\right)\right)+\sum_{1 \leq i<j \leq N} \frac{1}{\left|x_{i}-x_{j}\right|}+\sum_{1 \leq i<j \leq M} \frac{Z_{i} Z_{j}}{\left|R_{i}-R_{j}\right|}, \\
V_{R}(u)=-\sum_{j=1}^{M} \frac{Z_{j}}{\left|u-R_{j}\right|} .
\end{gathered}
$$

Its operator domain is the Sobolev space $H_{a}^{2}\left(\mathbb{R}^{3 N}, \mathbb{C}\right)$, and its quadratic form domain is $H_{a}^{1}\left(\mathbb{R}^{3 N}, \mathbb{C}\right)$. Throughout this paper, the subscript $a$ indicates that we consider wavefunctions $\Psi$ which are antisymmetric under interchanges of variables (expression of the Pauli exclusion principle):

$$
\forall \sigma \in S_{N}, \Psi\left(x_{1}, \ldots, x_{N}\right)=\epsilon(\sigma) \Psi\left(x_{\sigma(1)}, \ldots, x_{\sigma(N)}\right) .
$$

The quantum energy of the system in a state $\Psi \in H_{a}^{1}\left(\mathbb{R}^{3 N}, \mathbb{C}\right)$ is the associated quadratic form

$$
\mathcal{E}^{N}(R, \Psi)=\left\langle\Psi, H^{N}(R, Z) \Psi\right\rangle .
$$

We refer the reader to $[22,3]$ for a description of this model and a detailed explanation of the Born-Oppenheimer approximation. The properties of $H^{N}(R, Z)$ and its eigenfunctions are recalled below. For the sake of simplicity, we have neglected the spin and the dynamic of the nuclei, as in [25]. We would like also to mention that all the results in this paper can be adapted to the case of smeared nuclei, that is to say when the Coulomb potential $\frac{1}{\left|x-R_{i}\right|}$ is replaced by $\int_{\mathbb{R}^{3}} \frac{1}{\left|x-y-R_{i}\right|} d \mu_{i}(y)$ where $\mu_{i}$ is a probability measure on $\mathbb{R}^{3}$. Of course, $\frac{1}{\left|R_{i}-R_{j}\right|}$ has to be replaced by $\iint_{\mathbb{R}^{6}} \frac{1}{\left|R_{i}+y-\left(R_{j}+z\right)\right|} d \mu_{i}(y) d \mu_{j}(z)$. Remark that, in contrast to many other papers dealing with minimization, we work here with complex-valued wavefunctions, a hypothesis that plays a role in our results (see for instance Theorem 4 and the associated remarks).

$Z$ and $N$ being fixed such that $N \leq|Z|$, for each $R \in \Omega$, the problem

$$
E^{N}(R, Z)=\min \left\{\mathcal{E}^{N}(R, \Psi),\|\Psi\|_{L^{2}}=1\right\}
$$

has a solution $\Psi$, which is the ground state of the $N$ electrons interacting with the $M$ nuclei localized at the $R_{i}$.

For neutral molecules $(N=|Z|)$, it is also known that the problem

$$
E^{N}=\min _{R \in \Omega} E^{N}(R, Z)
$$


admits a solution [25], proving the stability of neutral molecules.

We shall assume that $(R, \Psi)$ and $\left(R^{\prime}, \Psi^{\prime}\right)$ are two local minima of $\mathcal{E}^{N}$. We then consider the classical mountain pass method

$$
c=\inf _{\gamma \in \Gamma} \max _{t \in[0 ; 1]} \mathcal{E}^{N}(\gamma(t))
$$

where

$$
\begin{gathered}
\Gamma=\left\{\gamma \in \mathcal{C}^{0}\left([0 ; 1], \Omega \times S H_{a}^{1}\left(\mathbb{R}^{3 N}\right)\right), \gamma(0)=(R, \Psi), \gamma(1)=\left(R^{\prime}, \Psi^{\prime}\right)\right\} \\
S H_{a}^{1}\left(\mathbb{R}^{3 N}\right)=\left\{\Psi \in H_{a}^{1}\left(\mathbb{R}^{3 N}\right),\|\Psi\|_{L^{2}}=1\right\}
\end{gathered}
$$

and want to show that $c$ is a critical value of $\mathcal{E}^{N}$.

As mentioned in the introduction, the physical interpretation of this minmax method is that paths $\gamma \in \Gamma$ represent an infinitely slow reaction leading from one minimum to the other. $c$ is thus interpreted as the lowest energy threshold for passing from $(R, \Psi)$ to $\left(R^{\prime}, \Psi^{\prime}\right)$.

In practice, the following definition is used

$$
c^{\prime}=\inf _{r \in \mathcal{R}} \max _{t \in[0 ; 1]} E^{N}(r(t), Z)
$$

where

$$
\mathcal{R}=\left\{r \in \mathcal{C}^{0}([0 ; 1], \Omega), r(0)=R, r(1)=R^{\prime}\right\} .
$$

As explained in the introduction, the function $R \mapsto E^{N}(R, Z)$ is continuous but not necessary differentiable and this is why we shall study the min-max method (1). However, it will be shown that in fact $c=c^{\prime}$.

\subsection{Properties of $H^{N}(R, Z)$}

Let us now recall some well-known facts about the spectrum of $H^{N}(R, Z)$. We introduce

$$
\lambda_{d}^{N}(R, Z)=\inf _{\operatorname{dim}(V)=d} \sup _{\substack{\Psi \in V,\|\Psi\|_{L^{2}}=1}}\langle\mathcal{H}(R, Z) \Psi, \Psi\rangle .
$$

In the sequel, we shall denote for all $d \geq 1$

$$
E^{0}(R, Z)=\lambda_{d}^{0}(R, Z):=\sum_{1 \leq i<j \leq M} \frac{Z_{i} Z_{j}}{\left|R_{i}-R_{j}\right|}, \quad \Sigma^{0}(R, Z)=+\infty .
$$

For a wavefunction $\Psi \in H_{a}^{1}\left(\mathbb{R}^{3}, \mathbb{C}\right)$, the electronic density and the electronic kinetic energy density are respectively defined by

$$
\begin{gathered}
\rho_{\Psi}(x)=N \int_{R^{3(N-1)}}\left|\Psi\left(x, x_{2}, \ldots, x_{N}\right)\right|^{2} d x_{2} \ldots d x_{N} \\
t_{\Psi}(x)=N \int_{R^{3(N-1)}}\left|\nabla \Psi\left(x, x_{2}, \ldots, x_{N}\right)\right|^{2} d x_{2} \ldots d x_{N} .
\end{gathered}
$$

We have brought together the main known results in the following 
Theorem 1. We assume $N \geq 1$. The following results are known:

1. (Self-adjointness [20]) $H^{N}(R, Z)$ is self-adjoint on $L_{a}^{2}\left(\mathbb{R}^{3 N}\right)$ with operator domain $H_{a}^{2}\left(\mathbb{R}^{3 N}\right)$ and quadratic form domain $H_{a}^{1}\left(\mathbb{R}^{3 N}\right)$.

2. We have [19]

$$
\begin{gathered}
\sigma_{\text {ess }}\left(H^{N}(R, Z)\right)=\left[\Sigma^{N}(R, Z) ;+\infty\right) \\
\forall d \geq 1, \lambda_{d}^{N}(R, Z) \leq \Sigma^{N}(R, Z) \leq \sum_{1 \leq i<j \leq M} \frac{Z_{i} Z_{j}}{\left|R_{i}-R_{j}\right|}
\end{gathered}
$$

3. (HVZ Theorem $[\mathbf{1 8}, \mathbf{3 2}, \mathbf{3 3}, \mathbf{1 9}])$ We have

$$
\Sigma^{N}(R, Z)=E^{N-1}(R, Z) .
$$

4. (Compactness below the essential spectrum) If $\lambda_{d}^{N}(R, Z)<\Sigma^{N}(R, Z)$, then $\lambda_{d}^{N}(R, Z)$ is an eigenvalue of finite multiplicity and in particular, there exists a $\Psi_{d} \in H_{a}^{2}\left(\mathbb{R}^{3 N}\right)$ such that

$$
H^{N}(R, Z) \Psi_{d}=\lambda_{d}^{N}(R, Z) \Psi_{d}
$$

It is locally lipschitz [21], i.e.

$$
\Psi_{d} \in \mathcal{C}^{0}\left(\mathbb{R}^{3 N}\right) \quad \text { and } \quad\left|\nabla \Psi_{d}\right| \in L_{l o c}^{\infty}\left(\mathbb{R}^{3 N}\right),
$$

and real analytic [13] on $U^{N} \backslash\left\{x_{i}=x_{j}\right\}$, where $U=\mathbb{R}^{3} \backslash\left\{R_{i}\right\}_{i=1}^{M}$. If $\rho_{d}$ is the associated electronic density, then [13]

$$
\rho_{d} \in \mathcal{C}^{\omega}(U) \cap \mathcal{C}^{0,1}\left(\mathbb{R}^{3}\right) .
$$

5. (Zhislin Theorem [33]) For positive and neutral molecules $N \leq|Z|$, then

$$
\lambda_{d}^{N}(R, Z)<\Sigma^{N}(R, Z)
$$

for all $d \geq 1$, so that

$$
\sigma\left(H^{N}(R, Z)\right)=\left\{\lambda_{1}^{N}(R, Z) \leq \cdots \leq \lambda_{d}^{N}(R, Z) \leq \cdots\right\} \cup\left[\Sigma^{N}(R, Z) ;+\infty\right) .
$$

6. (Negative molecules $[\mathbf{1 9}, \mathbf{2 3}]$ ) For negative molecules $N>|Z|$, there exists a $\delta$ such that $\lambda_{\delta}^{N}(R, Z)=\Sigma^{N}(R, Z)$, and $\delta=1$ when $N \geq 2|Z|+M$.

Note that the functions $\Sigma^{N}(R, Z)$ and $\lambda_{d}^{N}(R, Z)(d \geq 1)$ are continuous with respect to $R$.

\section{The results}

In this section, we present the results that we have obtained concerning the mountain pass method defined above. As mentioned, all the proofs are postponed to the next section. 


\subsection{The spectrum of a molecule that splits into pieces}

We begin the study by some general results about the spectrum and the behaviour of the eigenstates when the molecule splits into pieces, that is to say when $\left|R_{i}-R_{j}\right| \rightarrow+\infty$ for some $i$ and $j$. As mentioned above, this splitting of the molecule will be shown to be the main reason for the possible lack of compactness of Palais-Smale sequences.

Although only the case of ground states will be necessary for the sequel, we tackle here arbitrary excited states. In this section, we consider a positive or neutral molecule $(N \leq|Z|)$.

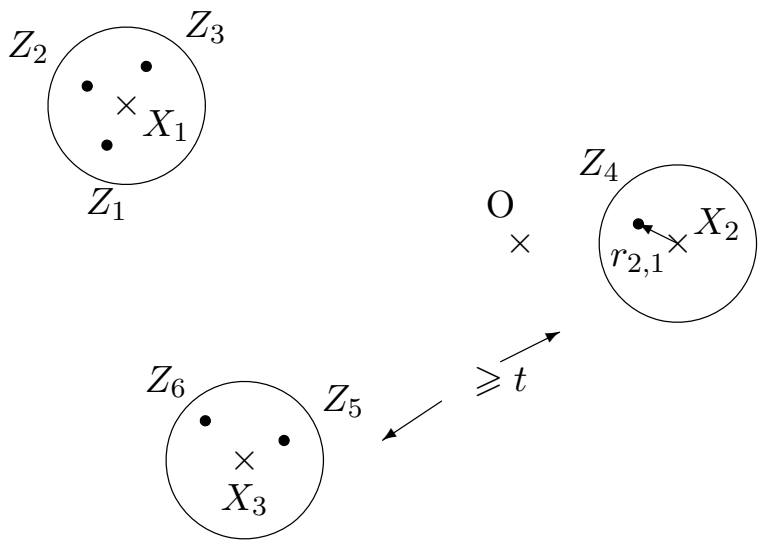

Figure 1: An example with $M=6, p=3, m_{1}=3, m_{2}=1$ and $m_{3}=2$.

We fix a $2 \leq p \leq M$ (number of pieces).

Let $X_{1}, \ldots, X_{p}: \mathbb{R}^{+} \mapsto \mathbb{R}^{3}$ be $p$ functions that satisfy $\left|X_{i}(t)-X_{j}(t)\right| \geq t$ for all $i \neq j$ and $t$ large enough. Let be $m=\left(m_{1}, \ldots, m_{p}\right) \in\left(\mathbb{N}^{*}\right)^{p}$ such that $\sum_{j=1}^{p} m_{j}=M$. We fix a positive constant $R_{0}$ and some $r_{j, k} \in \mathbb{R}^{3}$ and $z_{j, k} \in \mathbb{N}$ for $j=1, \ldots, p$ and $k=1, \ldots, m_{j}$ such that $|Z|:=\sum_{j=1}^{p} \sum_{k=1}^{m_{j}} z_{j, k} \geq N$, $\left|r_{j, k}\right| \leq R_{0}$ and $r_{j, k} \neq r_{j, l}$ when $k \neq l$. We then let

$$
\begin{gathered}
z_{j}=\left(z_{j, 1}, \ldots, z_{j, m_{j}}\right), \quad Z=\left(z_{1}, \ldots, z_{p}\right), \quad r_{j}=\left(r_{j, 1}, \ldots, r_{j, m_{j}}\right), \\
\tilde{r}_{j}(t)=\left(X_{j}(t)+r_{j, 1}, \ldots, X_{j}(t)+r_{j, m_{j}}\right), \quad R(t)=\left(\tilde{r}_{1}(t), \ldots, \tilde{r}_{p}(t)\right) .
\end{gathered}
$$

We also introduce

$$
\omega_{j}=\left\{\left(r_{k}\right) \in B\left(0, R_{0}\right)^{m_{j}}, r_{k_{1}} \neq r_{k_{2}} \text { if } k_{1}=k_{2}\right\}
$$

and

$$
\mathcal{U}(R)=\mathbb{R}^{3} \backslash\left(\bigcup_{j=1}^{p} \overline{B\left(X_{j}, R\right)}\right)
$$




\subsubsection{Spectrum and uniform exponential decay}

We have the following result:

Theorem 2 (Spectrum and uniform exponential decay). For all $1 \leq N \leq|Z|$ and all $d \geq 1$, we have

1. $\lim _{t \rightarrow+\infty} E^{N}(R(t), Z)=\min \left\{\sum_{j=1}^{p} E^{N_{j}}\left(r_{j}, z_{j}\right), N_{1}+\ldots+N_{p}=N\right\}$.

2. $\limsup _{t \rightarrow+\infty} \lambda_{d}^{N}(R(t), Z) \leq \min \left\{\sum_{j=1}^{p} \lambda_{\delta_{j}}^{N_{j}}\left(r_{j}, z_{j}\right), N_{1}+\ldots+N_{p}=N, \prod_{j=1}^{p} \delta_{j}=d\right\}$.

3. $\inf _{r_{j} \in \omega_{j}} \liminf _{t \rightarrow+\infty}\left(\Sigma^{N}(R(t), Z)-\lambda_{d}^{N}(R(t), Z)\right)>0$.

4. Let $\Psi_{R}$ be an eigenfunction associated to the eigenvalue $\lambda_{d}^{N}(R, Z)$, with associated densities $\rho_{R}$ and $t_{R}$. Then there exist positive constants $R_{1}, C$ and $\alpha$, depending only on $N, d, R_{0}, p$ such that

$$
\rho_{R}(x) \leq C \exp (-\alpha \delta(x)) \text { and } t_{R}(x) \leq C \exp (-\alpha \delta(x)) \quad \text { on } \mathcal{U}\left(R_{1}\right)
$$

where $\delta(x)=\min \left\{\left|x-X_{j}\right|, j=1, \ldots, d\right\}$.

The first part 1) identifies the limit of the ground state energy. This type of result is rather intuitive and classical. However, since we do not know a reference in this precise setting, a proof will be given in the next section.

The interpretation of the last part 4) is that if a neutral or positively charged molecule splits into parts, then for a fixed excited state, the electrons remain in the vicinity of the nuclei.

For the sake of simplicity, let us denote, for $r=\left(r_{1}, \ldots, r_{p}\right)$ and $z=\left(z_{1}, \ldots, z_{p}\right)$

$$
\Lambda_{d}^{N}(r, z):=\min \left\{\sum_{j=1}^{p} \lambda_{\delta_{j}}^{N_{j}}\left(r_{j}, z_{j}\right), N_{1}+\ldots+N_{p}=N, \prod_{j=1}^{p} \delta_{j}=d\right\} .
$$

\subsubsection{Behaviour of the wavefunctions, critical points at infinity}

Now that we have some bounds on the eigenvalues and the bottom of the essential spectrum, we want to prove a result describing the behaviour of the eigenfunctions. This will enable us to define the "critical points at infinity" of the model, a concept that was introduced by A. Bahri [2].

The right hand side of Theorem 2 - 1), or more generally an equality like

$$
c=\sum_{j=1}^{p} \lambda_{\delta_{j}}^{N_{j}}\left(r_{j}, z_{j}\right)
$$

is rather standard from P.-L. Lions' Concentration-Compactness point of view: when the molecule splits into pieces, then the energy of an electronic excited 
states becomes a sum of excited states energies of the pieces. In other words, a "critical point at infinity" would be a system constituted by $p$ molecules in some excited state, each being infinitely far from the others, so that the interactions between them vanish.

Let us consider a sequence $t_{n} \rightarrow+\infty$, some $r=\left(r_{1}, \ldots, r_{p}\right) \in \bigotimes_{j=1}^{p} \omega_{j}$, and denote by $R^{n}=R\left(t_{n}\right):=\left(X_{1}\left(t_{n}\right)+r_{1}, \ldots, X_{p}\left(t_{n}\right)+r_{p}\right), X_{j}^{n}=X_{j}\left(t_{n}\right)$. For the electronic density, such a configuration is then clearly obtained in the case of dichotomy, that is to say $\rho^{n} \simeq \sum_{j=1}^{p} \rho_{j}^{n}$ where $\rho_{j}^{n}$ is essentially supported in the vicinity of $X_{j}^{n}$ (see the exponential decay of Theorem 2). But the behaviour of the wavefunction $\Psi^{n}$ is less simple since these functions will not split into sum of functions, but into sums of antisymmetric tensor products of wavefunctions in lower dimensions. In other words, a simple way to represent these non interacting molecules in terms of the wavefunction is to take

$$
\Psi^{n}=\tau_{X_{1}^{n}} \cdot \psi_{1} \wedge \cdots \wedge \tau_{X_{p}^{n}} \cdot \psi_{p}
$$

where each $\psi_{j}$ is an eigenfunction of $H^{N_{j}}\left(r_{j}, z_{j}\right)$ associated to the eigenvalue $\lambda_{\delta_{j}}^{N_{j}}\left(r_{j}, z_{j}\right)$. We have used here the notation

$$
\tau_{v} \cdot \Psi\left(x_{1}, \ldots, x_{N}\right):=\Psi\left(x_{1}-v, \ldots, x_{N}-v\right)
$$

and we recall that the tensor product is defined for $\psi \in L_{a}^{2}\left(\mathbb{R}^{3 N_{1}}\right)$ and $\psi^{\prime} \in$ $L_{a}^{2}\left(\mathbb{R}^{3 N_{2}}\right)$ by

$$
\psi \wedge \psi^{\prime}\left(x_{1}, \ldots, x_{N_{1}+N_{2}}\right)=\frac{1}{\sqrt{N ! N_{1} ! N_{2} !}} \sum_{\sigma \in S_{N}} \varepsilon(\sigma) \psi\left(x_{\sigma}^{1}\right) \psi^{\prime}\left(x_{\sigma}^{2}\right) .
$$

where $x_{\sigma}^{1}:=\left(x_{\sigma(1)}, \ldots, x_{\sigma\left(N_{1}\right)}\right)$ and $x_{\sigma}^{2}=\left(x_{\sigma\left(N_{1}+1\right)}, \ldots, x_{\sigma(N)}\right)$.

With (4), one easily sees that $\rho^{n}=\sum_{j=1}^{p} \tau_{X_{j}^{n}} \cdot \rho_{j}$ with obvious notations, and that

$$
H^{N}\left(R^{n}, Z\right) \Psi^{n}-c \Psi^{n} \rightarrow 0
$$

in $L^{2}\left(\mathbb{R}^{3 N}\right)$ as $n \rightarrow+\infty$.

When a $\lambda_{\delta_{j}}^{N_{j}}\left(r_{j}, z_{j}\right)$ is degenerated, we can obtain the same behaviour by taking a wavefunction which is a sum of such antisymmetric tensor products

$$
\Psi^{n} \in \bigwedge_{j=1}^{p} \tau_{X_{j}^{n}} \cdot \operatorname{ker}\left(H^{N_{j}}\left(r_{j}, z_{j}\right)-\lambda_{\delta_{j}}^{N_{j}}\left(r_{j}, z_{j}\right)\right) .
$$

To simplify notations, we shall denote $\tau_{n} \cdot\left(\psi_{1} \wedge \cdots \wedge \psi_{p}\right):=\tau_{X_{1}^{n}} \cdot \psi_{1} \wedge \cdots \wedge \tau_{X_{p}^{n}} \cdot \psi_{p}$, so that $\Psi^{n}=\tau_{n} \cdot \Psi$ where $\Psi \in \bigwedge_{j=1}^{p} \operatorname{ker}\left(H^{N_{j}}\left(r_{j}, z_{j}\right)-\lambda_{\delta_{j}}^{N_{j}}\left(r_{j}, z_{j}\right)\right)$.

Suppose now that a molecule splits into two identical pieces: $r_{1}=r_{2}$ and $z_{1}=z_{2}$, and that $N_{1} \neq N_{2}$. At infinity, we shall obtain two molecules with the same configurations of the nuclei, but not the same number of electrons. Since there is no reason to distinguish the two states obtained by inverting the electrons between the two molecules, a wavefunction can be a sum of these two states with the same energies. We are thus led to introduce the following definition. 
Definition 1. Let $r^{n}=\left(r_{1}^{n}, \ldots, r_{p}^{n}\right) \in \bigotimes_{j=1}^{p} \omega_{j}$ be such that $r_{j}^{n} \rightarrow r_{j} \in \omega_{j}$, and $R^{n}=R\left(t_{n}\right):=\left(X_{1}\left(t_{n}\right)+r_{1}, \ldots, X_{p}\left(t_{n}\right)+r_{p}\right), X_{j}^{n}=X_{j}\left(t_{n}\right)$, for some $t_{n} \rightarrow+\infty$. Let $c$ be such that the set

$$
\mathcal{A}_{c}^{N}(r, z)=\left\{\left(N_{j}, \delta_{j}\right) \in\left(\mathbb{N}^{p}\right)^{2}, N_{1}+\cdots+N_{p}=N, \sum_{j=1}^{p} \lambda_{\delta_{j}}^{N_{j}}\left(r_{j}, z_{j}\right)=c\right\}
$$

is not empty. The sequence $\left(R^{n}, \Psi^{n}\right)$ in $\Omega \times S H_{a}^{1}\left(\mathbb{R}^{3 N}\right)$ converges to a critical point at infinity of energy $c$ if there exists some

$$
\Psi \in \sum_{\left(N_{j}, \delta_{j}\right) \in \mathcal{A}_{c}^{N}(r, z)}\left(\bigwedge_{j=1}^{p} \operatorname{ker}\left(H^{N_{j}}\left(r_{j}, z_{j}\right)-\lambda_{\delta_{j}}^{N_{j}}\left(r_{j}, z_{j}\right)\right)\right)
$$

such that

$$
\left\|\Psi^{n}-\tau_{n} \cdot \Psi\right\|_{H_{a}^{1}\left(\mathbb{R}^{3 N}\right)} \rightarrow 0
$$

To justify the term critical point, we remark that one can prove

Lemma 1. Let be $c$ and $\Psi$ that satisfy (5) and (6). Then

$$
\left(H^{N}\left(R^{n}, Z\right)-c\right)\left(\tau_{n} \cdot \Psi\right) \rightarrow 0
$$

in $L^{2}\left(\mathbb{R}^{3 N}\right)$.

Details will be given later on.

Saying differently, a critical point at infinity is a class

$$
\tau_{K}(r, \Psi)=\left\{\tau_{X_{1}, \ldots, X_{p}}(r, \Psi), X_{j} \in \mathbb{R}^{3},\left|X_{i}-X_{j}\right| \geq K\right\}
$$

where $\Psi \in \sum_{\left(N_{j}, \delta_{j}\right) \in \mathcal{A}_{c}^{N}(r, z)}\left(\bigwedge_{j=1}^{p} \operatorname{ker}\left(H^{N_{j}}\left(r_{j}, z_{j}\right)-\lambda_{\delta_{j}}^{N_{j}}\left(r_{j}, z_{j}\right)\right)\right), r_{j} \in \omega_{j}$ and $\tau_{X_{1}, \ldots, X_{p}}$ is defined on each $\Omega \times \bigwedge_{j=1}^{p} H_{a}^{1}\left(\mathbb{R}^{3 N_{j}}\right)$ by

$$
\begin{gathered}
\tau_{X_{1}, \ldots, X_{p}} \cdot r=\left(X_{1}+r_{1}, \cdots, X_{p}+r_{p}\right) \\
\tau_{X_{1}, \ldots, X_{p}} \cdot\left(\psi_{1} \wedge \cdots \wedge \psi_{p}\right)=\left(\tau_{X_{1}} \psi_{1}\right) \wedge \cdots \wedge\left(\tau_{X_{p}} \psi_{p}\right) .
\end{gathered}
$$

A sequence $\left(R^{n}, \Psi^{n}\right)$ converges to this critical point at infinity when there exists a $K_{n} \rightarrow+\infty$ such that

$$
\lim _{n \rightarrow+\infty} d\left[\left(R^{n}, \Psi^{n}\right) ; \tau_{K_{n}}(r, \Psi)\right]=0 .
$$

Let us now fix a sequence $t_{n} \rightarrow+\infty$ and some $r^{n}=\left(r_{1}^{n}, \ldots, r_{p}^{n}\right) \in \bigotimes_{j=1}^{p} \omega_{j}$ such that $r_{j}^{n} \rightarrow r_{j} \in \omega_{j}$, and denote $R^{n}=R\left(t_{n}\right), X_{j}^{n}=X_{j}\left(t_{n}\right)$. We then have the following result concerning the behaviour of the eigenfunctions 
Theorem 3. We assume $1 \leq N \leq|Z|$ and $d \geq 1$. Let $\left(\Psi^{n}\right)$ be a sequence of wavefunctions such that

$$
H^{N}\left(R^{n}, Z\right) \cdot \Psi^{n}=\lambda_{d}^{N}\left(R^{n}, Z\right) \cdot \Psi^{n} .
$$

Then, up to a subsequence, we have $\lim _{n \rightarrow+\infty} \lambda_{d}^{N}\left(R^{n}, Z\right):=c$ with

$$
\Lambda_{1}^{N}(r, z) \leq c=\sum_{j=1}^{p} \lambda_{\delta_{j}^{\prime}}^{N_{j}^{\prime}}\left(r_{j}, z_{j}\right) \leq \Lambda_{d}^{N}(r, z)
$$

for some $\left(N_{j}^{\prime}, \delta_{j}^{\prime}\right) \in \mathcal{A}_{c}^{N}(r, z)$, and $\left(R^{n}, \Psi^{n}\right)$ converges to a critical point at infinity of energy $c$.

\subsection{The mountain pass method: a general result}

Let us now come back to our mountain pass method, and consider again a neutral molecule $(N=|Z|)$. Recall that $(R, \Psi)$ and $\left(R^{\prime}, \Psi^{\prime}\right)$ are two local minima of $(R, \Psi) \mapsto \mathcal{E}^{N}(R, \Psi)$, and that $c$ and $c^{\prime}$ are defined by

$$
\begin{gathered}
c=\inf _{\gamma \in \Gamma} \max _{t \in[0 ; 1]} \mathcal{E}^{N}(\gamma(t)) \\
\Gamma=\left\{\gamma \in \mathcal{C}^{0}\left([0 ; 1], \Omega \times S H_{a}^{1}\left(\mathbb{R}^{3 N}\right)\right), \gamma(0)=(R, \Psi), \gamma(1)=\left(R^{\prime}, \Psi^{\prime}\right)\right\} . \\
c^{\prime}=\inf _{r \in \mathcal{R}} \max _{t \in[0 ; 1]} E^{N}(r(t), Z) \\
\mathcal{R}=\left\{r \in \mathcal{C}^{0}([0 ; 1], \Omega), r(0)=R, r(1)=R^{\prime}\right\} .
\end{gathered}
$$

The following result enables to identify $c$ in the case of lack of compactness:

Theorem 4. We assume $N=|Z|$. We have $c=c^{\prime}$. There exists a min-maxing sequence $\left(R^{n}, \Psi^{n}\right) \in \Omega \times H_{a}^{1}\left(\mathbb{R}^{3 N}\right)$ such that:

If $\sum_{i \neq j}\left|R_{i}^{n}-R_{j}^{n}\right|$ is bounded then, up to a translation, $\left(R^{n}, \Psi^{n}\right)$ converges strongly in $\Omega \times H_{a}^{1}\left(\mathbb{R}^{3 N}\right)$ to some critical point $(R, \Psi)$ of $\mathcal{E}^{N}$ such that

$$
H^{N}(R, Z) \cdot \Psi=c \cdot \Psi, \quad c=\lambda_{1}^{N}(R, Z) .
$$

If $\sum_{i \neq j}\left|R_{i}^{n}-R_{j}^{n}\right|$ is not bounded, there exists a $2 \leq p \leq M$, some $X_{j}^{n} \in \mathbb{R}^{3}$ with $j=1, \ldots, p$ and $a R_{0}>0$ such that, changing the indices if necessary, $R^{n}=\left(X_{1}^{n}+r_{1}^{n}, \ldots, X_{p}^{n}+r_{p}^{n}\right),\left\|r_{j}^{n}\right\| \leq R_{0}, Z=\left(z_{1}, \ldots, z_{p}\right)$, and $\lim _{n \rightarrow+\infty} \mid X_{i}^{n}-$ $X_{j}^{n} \mid=+\infty, r_{j}^{n} \rightarrow r_{j}$. Then

$$
c=\Lambda_{1}^{N}(r, z)=\min \left\{\sum_{j=1}^{p} E^{N_{j}}\left(r_{j}, z_{j}\right), N_{1}+\cdots+N_{p}=N\right\}
$$

and $\left(R^{n}, \Psi^{n}\right)$ converges up to a subsequence to a critical point at infinity of energy $c=\Lambda_{1}^{N}(r, z)$. 
As a consequence, in the non-compact case, the molecule splits into pieces, the electrons being shared among them and at their ground state. We also believe that the $r_{j}$ correspond to positions of the nuclei with a Morse index equal to 0 , but this is not necessary for the sequel.

This result should be seen as the first step towards concluding the existence of a critical point of energy $c$, by proving that the second case in Theorem 4 does not happen. Unfortunately, we met with serious difficulties when trying to solve this general problem. This is why the compactness will be shown in the next section for the special case of two interacting molecules with fixed nuclei.

Remark - Throughout this paper, we work with complex-valued wavefunctions $\Psi$. Although in other situations (minimization for instance) one often works with real-valued functions without any change, this is not the case here. In particular, the equality $c=c^{\prime}$ is very easily obtained in this setting, while one can prove that this is also true for real-valued wavefunctions, but for wellchosen ground states $\Psi$ and $\Psi^{\prime}$ only. See the proof for more details.

\subsection{Compactness in the case of two interacting molecules}

Now that we have identified the critical points at infinity for the mountain pass method, the next step is to show that min-maxing paths cannot approach these critical points. We study here the case of two interacting molecules with fixed nuclei. The parameters are then

- the distance between the two molecules (denoted by $\alpha$ in the sequel),

- the orientation of each molecule (represented by two rotations $u$ and $u^{\prime}$ ),

- the electronic wavefunction.

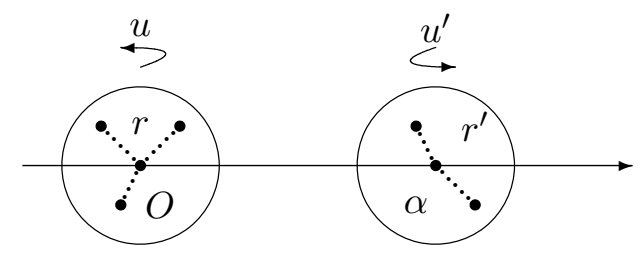

Figure 2: Two molecules with fixed nuclei.

So we consider $r=\left(r_{1}, \ldots, r_{m}\right) \in B\left(0, R_{0}\right)^{m}$ and $r^{\prime}=\left(r_{1}^{\prime}, \ldots, r_{m^{\prime}}^{\prime}\right) \in B\left(0, R_{0}\right)^{m^{\prime}}$ such that $r_{1}=r_{1}^{\prime}=0, r_{i} \neq r_{j}$ and $r_{i}^{\prime} \neq r_{j}^{\prime}$ for $i \neq j$, and some $z=\left(z_{1}, \ldots, z_{m}\right)$, $z^{\prime}=\left(z_{1}^{\prime}, \ldots, z_{m^{\prime}}^{\prime}\right)$. We denote by $Z=\left(z, z^{\prime}\right)$, and introduce

$$
R\left(\alpha, u, u^{\prime}\right)=\left(u \cdot r, \alpha \vec{v}+u^{\prime} \cdot r^{\prime}\right) .
$$


where $\vec{v}$ is a fixed vector of norm $1, \alpha \in \mathbb{R}$, and $u, u^{\prime}$ are rotations in $\mathbb{R}^{3}$. We have used the notation $u \cdot r=\left(u \cdot r_{1}, \ldots, u \cdot r_{m}\right)$. We suppose now that $N=|Z|$ and define

$$
\mathcal{E}^{N}\left(\alpha, u, u^{\prime}, \Psi\right):=\mathcal{E}^{N}\left(R\left(\alpha, u, u^{\prime}\right), \Psi\right) .
$$

In [25], it is proved that $\mathcal{E}^{N}$ admits a minimum on $\mathbb{R} \times\left(S \mathcal{O}_{3}(\mathbb{R})\right)^{2} \times S H_{a}^{1}\left(\mathbb{R}^{3 N}\right)$. As in the previous sections, we shall assume that $\mathcal{E}^{N}$ possesses two local minima $M$ and $M^{\prime}$. Up to a rotation of each molecule, we may suppose that $\alpha(M)>0$ and $\alpha\left(M^{\prime}\right)>0$. We then consider

$$
c=\inf _{\gamma \in \Gamma} \max _{t \in[0 ; 1]} \mathcal{E}^{N}(\gamma(t))
$$

where $\Gamma$ is the set of all the continuous functions $\gamma:[0 ; 1] \rightarrow X:=(0 ;+\infty) \times$ $\left(S \mathcal{O}_{3}(\mathbb{R})\right)^{2} \times S H_{a}^{1}\left(\mathbb{R}^{3 N}\right)$ such that $\gamma(0)=M$ and $\gamma(1)=M^{\prime}$.

\subsubsection{The mountain pass method}

We begin this section by stating a result which is the analogue of Theorem 4 in this special setting.

Theorem 5. We have

- either there exists a critical point $\left(\alpha, u, u^{\prime}, \Psi\right)$ of $\mathcal{E}^{N}$ on $X$, such that

$$
H^{N}\left(R\left(\alpha, u, u^{\prime}\right), Z\right) \cdot \Psi=c \cdot \Psi, \quad c=\lambda_{1}^{N}\left(R\left(\alpha, u, u^{\prime}\right), Z\right),
$$

- or

$$
c=\min \left\{E^{N_{1}}(r, z)+E^{N_{2}}\left(r^{\prime}, z^{\prime}\right), N_{1}+N_{2}=N\right\} .
$$

Roughly speaking, the non compactness of min-maxing sequences is related to the existence of two gradient lines going from a local minimum to some critical point at infinity of index 0 . The idea is that an "optimal path" has to follow these lines, and then to connect the two critical points at infinity. Since the molecule is split here into two independent parts, the problem is now to find two mountain pass paths connecting each configuration of the two molecules - two similar problems of lower dimension. When the position of the nuclei in each molecule is fixed, these paths can be obtained by only applying some rotations. In other words, the minima always belong to the same connected component and this is why the situation will be simpler in this setting. When the position is not fixed, even if we may assume the existence of such paths (by induction), the situation is much more complicated and we hope to come back to this more general issue in the future.

The proof of Theorem 5 is very similar to the one of Theorem 4, and will be ommitted.

In order to prove that the second case in Theorem 5 does not happen, we need some information on the directions on which the energy decreases near the critical points at infinity. We shall thus need an expansion of the interaction energy between the two molecules when $\alpha$ grows. The terms involving in these expansion are classical. Let us first recall the definitions of the first multipoles. 
Definition 2. Let be $R=\left(R_{1}, \ldots, R_{M}\right) \in \Omega, Z=\left(Z_{1}, \ldots, Z_{M}\right)$, and $\rho \in$ $L^{1}\left(\mathbb{R}^{3}\right) \cap \mathcal{S}\left(\mathbb{R}^{3} \backslash\left\{R_{j}\right\}\right)$ a non negative function such that $\int_{\mathbb{R}^{3}} \rho=N>0$. Then

1. the total density of charge $i s$ the measure $\tilde{\rho}:=\rho-\sum_{j=1}^{M} Z_{j} \delta_{R_{j}}$. The total charge is $q:=\int_{\mathbb{R}^{3}} \tilde{\rho}=N-|Z|$,

2. the dipole moment is the vector $P:=\int_{\mathbb{R}^{3}} x \tilde{\rho}(x) d x=\int_{\mathbb{R}^{3}} x \rho(x) d x-$ $\sum_{j=1}^{M} Z_{j} R_{j}$,

3. the quadrupole moment is the matrix $Q:=\int_{\mathbb{R}^{3}}\left(x x^{T}-\frac{1}{3}|x|^{2} I\right) \tilde{\rho}(x) d x$.

When $\rho$ is the electronic density associated to some eigenstate $\Psi$, we shall use the notations $\rho_{\Psi}, \tilde{\rho}_{\Psi}, P_{\Psi}$ and $Q_{\Psi}$.

This multipoles will be used in the expansion of the interaction energy. To illustrate this point, we give here the following

Lemma 2. We assume that $N_{1}$ and $N_{2}$ are such that $N_{1}+N_{2}=N, E^{N_{1}}(r, z)<$ $\Sigma^{N_{1}}(r, z)$ and $E^{N_{2}}\left(r^{\prime}, z^{\prime}\right)<\Sigma^{N_{2}}\left(r^{\prime}, z^{\prime}\right)$. Let $\psi_{1}$ and $\psi_{2}$ be two ground states of respectively $H^{N_{1}}(r, z)$ and $H^{N_{2}}\left(r^{\prime}, z^{\prime}\right)$. Denoting

$$
\Psi\left(\alpha, u, u^{\prime}\right)=\left(u \cdot \psi_{1}\right) \wedge\left(\tau_{\alpha \vec{v}} \cdot u^{\prime} \cdot \psi_{2}\right),
$$

we have

$$
\begin{array}{r}
\mathcal{E}^{N}\left(R\left(\alpha, u, u^{\prime}\right), \Psi\left(\alpha, u, u^{\prime}\right)\right)=E^{N_{1}}(r, z)+E^{N_{2}}\left(r^{\prime}, z^{\prime}\right) \\
+\frac{q_{1} q_{2}}{\alpha}+q_{2} \frac{u P_{1} \cdot \vec{v}}{\alpha^{2}}-q_{1} \frac{u^{\prime} P_{2} \cdot \vec{v}}{\alpha^{2}}+\frac{\left(u P_{1}\right) \cdot\left(u^{\prime} P_{2}\right)-3\left(u P_{1} \cdot \vec{v}\right)\left(u^{\prime} P_{2} \cdot \vec{v}\right)}{\alpha^{3}} \\
+\frac{3\left(q_{2} u Q_{1} u^{T}+q_{1} u^{\prime} Q_{2} u^{\prime T}\right) v \cdot v}{2 \alpha^{3}}+O\left(\frac{1}{\alpha^{4}}\right)
\end{array}
$$

for all $u, u^{\prime} \in S \mathcal{O}_{3}(\mathbb{R})$ and when $\alpha$ goes to $+\infty$.

In this result, $q_{k}, P_{k}$ and $Q_{k}$ are respectively the total charge, the dipole and the quadrupole moment associated to the electronic densities $\rho_{k}$ of the states $\psi_{k}$.

The terms of this expansion can be interpreted respectively as the energies of the molecules, and the interaction energy between them, which decomposes into the charge/charge $(1 / \alpha)$, dipole/charge $\left(1 / \alpha^{2}\right)$, dipole/dipole and charge/quadrupole $\left(1 / \alpha^{3}\right)$ terms.

We are now able to state our main compactness results. As mentioned above, we had to add some hypothesis about the molecules "at infinity", concerning their multipoles in their ground state.

\subsubsection{The case of charged molecules at infinity}

Our first result will concern the case of monopoles at infinity, that is to say when the molecules are charged. 
Theorem 6 (Charged molecules at infinity). Let us assume that

$$
E^{N_{1}}(r, z)+E^{N_{2}}\left(r^{\prime}, z^{\prime}\right)=\min \left\{E^{n_{1}}(r, z)+E^{n_{2}}\left(r^{\prime}, z^{\prime}\right), n_{1}+n_{2}=N\right\}
$$

for some $N_{1}$ and $N_{2}$ with $\left(N_{1}-|z|\right)\left(N_{2}-\left|z^{\prime}\right|\right) \neq 0$.

Then the case 2) in Theorem 5 does not happen. Therefore $c$ is a critical value of $\mathcal{E}^{N}$ on $X$.

Remark - By (9), we have for instance

$$
\mu:=E^{N_{1}}(r, z)-E^{|z|}(r, z)<E^{\left|z^{\prime}\right|}\left(r^{\prime}, z^{\prime}\right)-E^{N_{2}}\left(r^{\prime}, z^{\prime}\right):=\mu^{\prime}
$$

for some $N_{1}, N_{2}$ such that $N_{1}+N_{2}=N$ and $N_{1}<|z|$. This can be viewed as a comparison between oxydo-reduction potentials. So (9) will be probably true if one molecule is a oxydant and the other is a reductor.

\subsubsection{The case of neutral molecules with dipole moments at infinity}

If the two molecules at infinity are neutral, the first term involving in the expansion of the interaction energy is the dipole/dipole term. This is why we shall now consider the case of molecules that possess some dipole moment in their ground state (experiment suggests that this is the case for every non symmetric molecule).

Let us introduce the following definition

Definition 3. Let be $R=\left(R_{1}, \ldots, R_{M}\right) \in \Omega, Z=\left(Z_{1}, \ldots, Z_{M}\right)$ and $N>0$ such that $\lambda_{1}^{N}(R, Z)<\Sigma^{N}(R, Z)$. We shall say that the molecule $(R, Z, N)$ possesses a dipole moment at its ground state if $P_{\Psi} \neq 0$ for all ground state $\Psi$.

Since $V:=\operatorname{ker}\left(H^{N}(R, Z)-E^{N}(R, Z)\right)$ is finite dimensional, let us notice that this implies $\min \left\{\left|P_{\Psi}\right|, \Psi \in V,\|\Psi\|_{L^{2}}=1\right\}>0$.

We then have the following result:

Theorem 7 (Neutral molecules with dipole moments at infinity).

Let us assume that

(H1) $E^{|z|}(r, z)+E^{\left|z^{\prime}\right|}\left(r^{\prime}, z^{\prime}\right)<E^{N_{1}}(r, z)+E^{N_{2}}\left(r^{\prime}, z^{\prime}\right)$ for all $N_{1}, N_{2}$ such that $N_{1}+N_{2}=N$ and $\left(N_{1}-|z|\right)\left(N_{2}-\left|z^{\prime}\right|\right) \neq 0$,

(H2) the two molecules $(r, z,|z|)$ and $\left(r^{\prime}, z^{\prime},\left|z^{\prime}\right|\right)$ possess a dipole moment at their ground state,

(H3) $E^{|z|}(r, z)$ or $E^{\left|z^{\prime}\right|}\left(r^{\prime}, z^{\prime}\right)$ is non-degenerated.

Then the case 2) in Theorem 5 does not happen. Therefore $c$ is a critical value of $\mathcal{E}^{N}$ on $X$.

Remark - (H3) is a purely mathematical restriction that simplifies the proof.

Let us explain the general idea of the proof. Recall that the dipole/dipole interaction energy can be written $F\left(P, P^{\prime}\right) / \alpha^{3}$ (see Lemma 2). It is shown in 
Appendix 2 that the critical points of $F$ which have a non-negative energy have a Morse index which is at least one. If a path approaches a critical point at infinity then, to pull down the energy along the path, one may use either the rotations of the molecules if the dipole/dipole interaction energy is positive (thanks to this Morse index information on $F$ ), or the distance between them if it is negative (because $\alpha \mapsto F\left(P, P^{\prime}\right) / \alpha^{3}$ is then increasing). This is why min-maxing paths do not approach the critical points at infinity, and give thus a compact Palais-Smale sequence. Obviously, this general idea does not suffice to lead the proof and there are some other difficulties (essentially due to the complexity of the model) that are explicited in the next section.

Remark - This general information on the Morse index is probably true for the others multipoles interaction energies, a fact that could be used to treat the general case.

\section{Proofs}

\subsection{Proof of Theorems 2 and 3}

\subsubsection{Preliminaries}

We shall use the following lemma, which is an adaptation of results in $[15,16$, 11, 12, 13], and which is proved in Appendix 1.

Lemma 3. Let $\Psi_{R}$ be an eigenfunction associated to the eigenvalue $\lambda_{d}^{N}(R, Z)$ and $\rho_{R}$ be the electronic density. We introduce $\epsilon_{R}=\Sigma^{N}(R, Z)-\lambda_{d}^{N}(R, Z)$. Then

1. $\rho_{R}$ satisfies the inequation

$$
-\frac{1}{2} \Delta \rho_{R}+V_{R} \rho_{R}+\epsilon_{R} \rho_{R} \leq 0 .
$$

2. With $R_{1}(\epsilon):=\max \left(R_{0}+1, R_{0}+\frac{2 N p}{\epsilon}\right)$ and $C(\epsilon):=\cup_{j=1}^{p}\left\{x,\left|x-X_{j}\right|=\right.$ $\left.R_{1}(\epsilon)\right\}$, and if $r>2 R_{1}\left(\epsilon_{R}\right)$, then we have

$$
\begin{aligned}
\rho_{R}(x) & \leq\left\|\rho_{R}\right\|_{L^{\infty}\left(C\left(\epsilon_{R}\right)\right)} \sum_{j=1}^{p} e^{-\sqrt{\epsilon_{R} / p}\left(\left|X_{j}-x\right|-R_{1}\left(\epsilon_{R}\right)\right)} \\
& \leq p\left\|\rho_{R}\right\|_{L^{\infty}\left(C\left(\epsilon_{R}\right)\right)} e^{-\sqrt{\epsilon_{R} / p}\left(\delta(x)-R_{1}\left(\epsilon_{R}\right)\right)} \\
& \leq M e^{-\sqrt{\epsilon_{R} / p}\left(\delta(x)-R_{1}\left(\epsilon_{R}\right)\right)}
\end{aligned}
$$

on $\mathcal{U}\left(R_{1}\left(\epsilon_{R}\right)\right)$, where $\delta(x)=\min \left\{\left|x-X_{j}\right|, j=1, \ldots, d\right\}$, and $M=$ $M\left(p, N, R_{1}\left(\epsilon_{R}\right)\right)$.

The explicit bound (11) has been written in order to show the dependence of all the constants with regard to $\epsilon_{R}$. It is clearly not optimal. It shows a non-isotropic exponential decay of the electronic density, which will be uniform if $\epsilon_{R} \nrightarrow 0$. This type of bounds is studied in the work of Agmon [1] and we 
do not know if one can use his formalism to obtain the same result. Isotropic exponential bounds for $N$-body eigenfunctions are frequently seen in the literature, but surprising is the fact that such non-isotropic bounds has not yet been noticed.

The next two lemmas will be useful to prove the exponential decay of Theorem 2 .

Lemma 4. For all $\alpha>0$, there exists a constant $M=M\left(\alpha, N, R_{0}\right)$ such that

$$
t_{R}(x) \leq M \int_{B(x, \alpha)} \rho_{R}(y) d y
$$

on $\mathcal{U}\left(R_{0}+1 / 2+\alpha\right)$.

Proof of Lemma 4 - see [16].

Lemma 5. For all $j=1, \ldots, p, d \geq 1$ and $n \leq\left|z_{j}\right|$, we have

$$
\inf _{r_{j} \in \omega_{j}}\left(\Sigma^{n}\left(r_{j}, z_{j}\right)-\lambda_{d}^{n}\left(r_{j}, z_{j}\right)\right)>0 .
$$

Proof - We have

$$
\Sigma^{n}\left(r_{j}, z_{j}\right)-\lambda_{d}^{n}\left(r_{j}, z_{j}\right)=\tilde{\Sigma}^{n}\left(r_{j}, z_{j}\right)-\tilde{\lambda}_{d}^{n}\left(r_{j}, z_{j}\right)
$$

where $\tilde{\lambda}_{d}^{n}\left(r_{j}, z_{j}\right)$ and $\tilde{\Sigma}^{n}\left(r_{j}, z_{j}\right)$ are the $d^{t h}$ eigenvalue and the bottom of the essential spectrum of the Hamiltonian with the nuclei interaction removed

$$
\tilde{H}^{n}\left(r_{j}, z_{j}\right)=\sum_{i=1}^{n}\left(-\frac{1}{2} \Delta_{x_{i}}+V_{r_{j}}\left(x_{i}\right)\right)+\sum_{1 \leq i<j \leq n} \frac{1}{\left|x_{i}-x_{j}\right|} .
$$

By Zhislin's Theorem, it is known that

$$
\tilde{\Sigma}^{n}\left(r_{j}, z_{j}\right)-\tilde{\lambda}_{d}^{n}\left(r_{j}, z_{j}\right)>0
$$

for all $r_{j} \in \overline{\omega_{j}}$ and, since this function is continuous with regard to $r_{j}$,

$$
\inf _{r_{j} \in \omega_{j}}\left(\Sigma^{n}\left(r_{j}, z_{j}\right)-\lambda_{d}^{n}\left(r_{j}, z_{j}\right)\right)>0 .
$$

In the next result, we use both HVZ and Zhislin's Theorems. This lemma will be useful in the proof of Theorem 2 to construct test functions.

Lemma 6. If the minimum

$$
\min \left\{\sum_{j=1}^{p} \lambda_{\delta_{j}}^{N_{j}}\left(r_{j}, z_{j}\right), N_{1}+\ldots+N_{p}=N, \prod_{j=1}^{p} \delta_{j}=d\right\} .
$$

is attained for $N_{1}, \ldots, N_{j}$ and $\delta_{1}, \ldots, \delta_{p}$, then necessarily

$$
\lambda_{\delta_{j}}^{N_{j}}\left(r_{j}, z_{j}\right)<\Sigma_{\delta_{j}}^{N_{j}}\left(r_{j}, z_{j}\right)
$$

for all $j=1, \ldots, p$. 
Proof of Lemma 6 - Remark that by definition $\lambda_{\delta_{j}}^{0}\left(r_{j}, z_{j}\right)<\Sigma^{0}\left(r_{j}, z_{j}\right)=$ $+\infty$ for all $\delta_{j}$. We argue by contradiction and suppose that there exists a $k$ such that $N_{k}>0$ and $\lambda_{\delta_{k}}^{N_{k}}\left(r_{k}, z_{k}\right)=\Sigma^{N_{k}}\left(r_{k}, z_{k}\right)=E^{N_{k}-1}\left(r_{k}, z_{k}\right)$. Theorem 1 implies $N_{k} \geq\left|z_{k}\right|+1$. Since $\sum_{j=1}^{p}\left(N_{k}-\left|z_{k}\right|\right)=N-|Z| \leq 0$, there exists a $l \neq k$ such that $N_{l}<\left|z_{l}\right|$. We then let $\delta_{j}^{\prime}=\delta_{j}, N_{j}^{\prime}=N_{j}$ for $j \notin\{k, l\}, \delta_{k}^{\prime}=1$, $N_{k}^{\prime}=N_{k}-1, \delta_{l}^{\prime}=\delta_{k} \delta_{l}$, and $N_{l}^{\prime}=N_{l}+1$. We obtain

$$
\begin{aligned}
\sum_{j=1}^{p} \lambda_{\delta_{j}}^{N_{j}}\left(r_{j}, z_{j}\right)-\sum_{j=1}^{p} \lambda_{\delta_{j}^{\prime}}^{N_{j}^{\prime}}\left(r_{j}, z_{j}\right) & =\lambda_{\delta_{l}}^{N_{l}}\left(r_{l}, z_{l}\right)-\lambda_{\delta_{l}^{\prime}}^{N_{l}+1}\left(r_{l}, z_{l}\right) \\
& \geq E^{N_{l}}\left(r_{l}, z_{l}\right)-\lambda_{\delta_{l}^{\prime}}^{N_{l}+1}\left(r_{l}, z_{l}\right) \\
& =\Sigma^{N_{l}+1}\left(r_{l}, z_{l}\right)-\lambda_{\delta_{l}^{\prime}}^{N_{l}+1}\left(r_{l}, z_{l}\right) \\
& >0
\end{aligned}
$$

since $N_{l}+1 \leq\left|z_{l}\right|$ (Zhislin Theorem), which is a contradiction.

\subsubsection{Proof of Theorem 2}

We are now able to prove Theorem 2.

We first prove 2). Suppose that the right hand side is attained for some $N_{1}, \ldots, N_{j}$ and $\delta_{1}, \ldots, \delta_{p}$ such that $N_{1}+\ldots+N_{p}=N$ and $\prod_{j=1}^{p} \delta_{j}=p$. For the sake of simplicity, we may assume that $N_{j}>0$ for all $j=1, \ldots, p$. By Lemma 6 and Theorem 1, there exist eigenfunctions $\Psi_{j}^{k} \in L_{a}^{2}\left(\mathbb{R}^{3 N_{j}}\right)$ satisfying

$$
H^{N_{j}}\left(r_{j}, z_{j}\right) \Psi_{j}^{k}=\lambda_{k}^{N_{j}}\left(r_{j}, z_{j}\right) \Psi_{j}^{k}, \quad \int_{\mathbb{R}^{3 N_{j}}} \Psi_{j}^{k} \Psi_{j}^{l}=\delta_{k l}
$$

for all $j=1, \ldots, p$ and $k=1, \ldots, \delta_{j}$. If

$$
V_{j}=\operatorname{span}\left(\Psi_{j}^{k}, k=1, \ldots, \delta_{j}\right) \subset L_{a}^{2}\left(\mathbb{R}^{3 N_{j}}\right)
$$

then we have

$$
\max _{\Psi \in V_{j},\|\Psi\|_{L}^{2}=1}\left\langle H^{N_{j}}\left(r_{j}, z_{j}\right) \Psi, \Psi\right\rangle=\lambda_{\delta_{j}}^{N_{j}}\left(r_{j}, z_{j}\right) .
$$

We now consider a sequence $t_{n} \rightarrow+\infty$ such that $\lim _{n \rightarrow+\infty} \lambda_{d}^{N}\left(R\left(t_{n}\right), Z\right)=$ $\lim \sup _{t \rightarrow+\infty} \lambda_{d}^{N}(R(t), Z)$. If $\Psi \in L^{2}\left(\mathbb{R}^{3 N}\right)$, we introduce

$$
\begin{gathered}
\Psi_{j}^{k, n}=\tau_{X_{j}\left(t_{n}\right)} \cdot \Psi_{j}^{k} \\
\tilde{V}_{j}^{n}=\operatorname{span}\left(\Psi_{j}^{k, n}, k=1, \ldots, \delta_{j}\right) \subset L_{a}^{2}\left(\mathbb{R}^{3 N_{j}}\right) .
\end{gathered}
$$

(we recall that $\tau_{v}$ is the translation by $v$ ).

Now, let be

$$
W_{n}=\tilde{V}_{1}^{n} \wedge \cdots \wedge \tilde{V}_{p}^{n}=\operatorname{span}\left(\Psi_{1}^{k_{1}, n} \wedge \cdots \wedge \Psi_{p}^{k_{p}, n}, 1 \leq k_{j} \leq \delta_{j}\right)
$$

which is a space of dimension $\prod_{j=1}^{d} \delta_{j}=d$. If

$$
\Psi=\sum_{1 \leq k_{j} \leq \delta_{j}} c_{k_{1}, \ldots, k_{p}} \Psi_{1}^{k_{1}, n} \wedge \cdots \wedge \Psi_{p}^{k_{p}, n} \in W_{n}
$$


and $\sum\left|c_{k_{1}, \ldots, k_{p}}\right|^{2}=1$, we have

$$
\left\langle H^{N}(R, Z) \Psi, \Psi\right\rangle=\sum_{j=1}^{p} \sum_{1 \leq k_{j} \leq \delta_{j}}\left|c_{k_{1}, \ldots, k_{p}}\right|^{2}\left\langle H^{N_{j}}\left(r_{j}, z_{j}\right) \Psi_{j}^{k_{j}, n}, \Psi_{j}^{k_{j}, n}\right\rangle+e_{n}
$$

where $e_{n}$ is the interaction energy between the $p$ molecules. It is the sum of three terms

$$
e_{n}=e_{n}^{1}+e_{n}^{2}+e_{n}^{3} .
$$

$e_{n}^{1}$ is the interaction between electrons in different molecules, and contains terms like

$$
\iint \frac{\left.\left(\Psi_{j_{1}}^{k_{j_{1}}} \Psi_{j_{1}}^{k_{j_{1}}^{\prime}}\right)(x, \ldots) \Psi_{j_{2}}^{k_{j_{2}}} \Psi_{j_{2}}^{k_{j_{2}}^{\prime}}\right)(y, \ldots)}{\left|x-y+X_{j_{2}}\left(t_{n}\right)-X_{j_{1}}\left(t_{n}\right)\right|} d x d y
$$

with $j_{1} \neq j_{2} . \quad e_{n}^{2}$ is the interaction between electrons and nuclei of different molecules, and contains terms like

$$
\iint \frac{z_{j_{2}, i}\left(\Psi_{j_{1}}^{k_{j_{1}}} \Psi_{j_{1}}^{k_{j_{1}}^{\prime}}\right)(x, \ldots)}{\left|x-r_{j_{2}, i}+X_{j_{2}}\left(t_{n}\right)-X_{j_{1}}\left(t_{n}\right)\right|} d x d y
$$

with $j_{1} \neq j_{2}$. Finally, $e_{n}^{3}$ is the interaction between nuclei of different molecules

$$
e_{n}^{3}=\sum_{j_{1}<j_{2}} \sum_{1 \leq k_{j} \leq m_{j}} \frac{z_{j_{1}, k_{j_{1}}} z_{j_{2}, k_{j_{1}}}}{\left|r_{j_{1}, k_{j_{1}}}-r_{j_{2}, k_{j_{2}}}+X_{j_{2}}\left(t_{n}\right)-X_{j_{1}}\left(t_{n}\right)\right|} .
$$

It is now easy to see that each of this term tends to 0 as $n \rightarrow+\infty$.

By definition, we have

$$
\begin{aligned}
\lambda_{d}^{N}(R, Z) & \leq \max _{\sum\left|c_{k_{1}, \ldots, k_{p}}\right|^{2}=1}\left\langle H^{N}(R, Z) \Psi, \Psi\right\rangle \\
& \leq \sum_{j=1}^{p} \max _{\sum\left|c_{k_{1}, \ldots, k_{p}}\right|^{2}=1} \sum_{1 \leq k_{j} \leq \delta_{j}}\left|c_{k_{1}, \ldots, k_{p}}\right|^{2} \lambda_{k_{j}}^{N_{j}}\left(r_{j}, z_{j}\right)+\max e_{n} \\
& \leq \sum_{j=1}^{p} \lambda_{\delta_{j}}^{N_{j}}\left(r_{j}, z_{j}\right)+\max e_{n} .
\end{aligned}
$$

We may now pass to the limit as $n \rightarrow+\infty$ in this inequality and obtain the bound

$$
\limsup _{t \rightarrow+\infty} \lambda_{d}^{N}(R(t), Z) \leq \min \left\{\sum_{j=1}^{p} \lambda_{\delta_{j}}^{N_{j}}\left(r_{j}, z_{j}\right), N_{1}+\ldots+N_{p}=N, \prod_{j=1}^{p} \delta_{j}=d\right\} .
$$

We then prove simultaneously 1) 3) 4) by induction on $N=1, \ldots,|Z|$.

For $N=1$, it is known that

$$
\Sigma^{1}(R, Z)=E^{0}(R, Z)=\sum_{1 \leq i<j \leq M} \frac{Z_{i} Z_{j}}{\left|R_{i}-R_{j}\right|},
$$


and so

$$
\lim _{t \rightarrow+\infty} \Sigma^{1}(R(t), Z)=\sum_{j=1}^{p} \sum_{1 \leq k<l \leq m_{j}} \frac{z_{j, k} z_{j, l}}{\left|r_{j, k}-r_{j, l}\right|}=\sum_{j=1}^{p} E^{0}\left(r_{j}, z_{j}\right) .
$$

As a consequence

$$
\begin{aligned}
& \liminf _{t \rightarrow+\infty}\left(\Sigma^{1}(R(t), Z)-\lambda_{d}^{1}(R(t), Z)\right) \\
& \geq \sum_{j=1}^{p} E^{0}\left(r_{j}, z_{j}\right)-\left(\lambda_{d}^{1}\left(r_{1}, z_{1}\right)+\sum_{j=2}^{p} E^{0}\left(r_{j}, z_{j}\right)\right) \\
& \quad=E^{0}\left(r_{1}, z_{1}\right)-\lambda_{d}^{1}\left(r_{1}, z_{1}\right)=\Sigma^{1}\left(r_{1}, z_{1}\right)-\lambda_{d}^{1}\left(r_{1}, z_{1}\right)
\end{aligned}
$$

and

$$
\inf _{r_{i} \in \omega_{i}} \liminf _{t \rightarrow+\infty}\left(\Sigma^{1}(R(t), Z)-\lambda_{d}^{1}(R(t), Z)\right) \geq \inf _{r_{1} \in \omega_{1}}\left(\Sigma^{1}\left(r_{1}, z_{1}\right)-\lambda_{d}^{1}\left(r_{1}, z_{1}\right)\right)>0
$$

by lemma 5 . The uniform exponential decay is then a consequence of lemmas 3 and 4.

Let $t_{n} \rightarrow+\infty$ be such that $\lim _{n \rightarrow+\infty} E^{1}\left(R\left(t_{n}\right), Z\right)=\liminf _{t \rightarrow+\infty} E^{1}(R(t), Z)$, and $\phi^{n} \in L^{2}\left(\mathbb{R}^{3}\right)$ be such that

$$
H^{1}\left(R\left(t_{n}\right), Z\right) \phi^{n}=E^{1}\left(R\left(t_{n}\right), Z\right) \phi^{n} .
$$

By the uniform exponential decay, we may write $\phi^{n}=\sum_{j=1}^{p} \phi_{j}^{n}+\alpha_{n}$ where $\operatorname{supp}\left(\phi_{j}^{n}\right) \subset B\left(X_{j}, r_{n} / 3\right)$, and $\left\|\alpha_{n}\right\|_{H^{1}} \rightarrow 0$. Then

$$
\left\langle H^{1}\left(R\left(t_{n}\right), Z\right) \phi^{n}, \phi^{n}\right\rangle=\sum_{j=1}^{p}\left\langle\tilde{H}^{1}\left(r_{j}, z_{j}\right) \phi_{j}^{n}, \phi_{j}^{n}\right\rangle+\sum_{j=1}^{p} \sum_{1 \leq k<l \leq m_{j}} \frac{z_{j, k} z_{j, l}}{\left|r_{j, k}-r_{j, l}\right|}+e_{n}
$$

where $\tilde{H}$ is the Hamiltonian with the nuclei interaction removed, and $e_{n} \rightarrow 0$. We have

$$
\begin{aligned}
\sum_{j=1}^{p}\left\langle\tilde{H}^{1}\left(r_{j}, z_{j}\right) \phi_{j}^{n}, \phi_{j}^{n}\right\rangle \geq \sum_{j=1, \ldots, p} \tilde{E}^{1}\left(r_{j}, z_{j}\right)\left\|\phi_{j}^{n}\right\|_{L^{2}}^{2} & \\
& \geq \min _{j=1, \ldots, p} \tilde{E}^{1}\left(r_{j}, z_{j}\right)\left(\sum_{j=1, \ldots, p}\left\|\phi_{j}^{n}\right\|_{L^{2}}^{2}\right)
\end{aligned}
$$

so

$$
\begin{aligned}
\left\langle H^{1}\left(R\left(t_{n}\right), Z\right) \phi^{n}, \phi^{n}\right\rangle & \geq \min _{j=1, \ldots, p} \tilde{E}^{1}\left(r_{j}, z_{j}\right)+\sum_{j=1}^{p} \sum_{1 \leq k<l \leq m_{j}} \frac{z_{j, k} z_{j, l}}{\left|r_{j, k}-r_{j, l}\right|} \\
& =\min \left\{\sum_{j=1}^{p} E^{N_{j}}\left(r_{j}, z_{j}\right), N_{1}+\ldots+N_{p}=1\right\}
\end{aligned}
$$


and finally

$$
\lim _{t \rightarrow+\infty} E^{1}(R(t), Z)=\min \left\{\sum_{j=1}^{p} E^{N_{j}}\left(r_{j}, z_{j}\right), N_{1}+\ldots+N_{p}=1\right\} .
$$

Let us now assume that 1) 3) 4) have been proved for $N-1<|Z|$. We have $\Sigma^{N}(R, Z)=\lambda_{1}^{N-1}(R, Z)$ so

$$
\begin{aligned}
\lim _{t \rightarrow+\infty} \Sigma^{N}(R(t), Z) & =\min \left\{\sum_{j=1}^{p} E^{N_{j}}\left(r_{j}, z_{j}\right), N_{1}+\ldots+N_{p}=N-1\right\} \\
& =\sum_{j=1}^{p} E^{N_{j}}\left(r_{j}, z_{j}\right)
\end{aligned}
$$

for some $N_{1}, \ldots, N_{p}$. But $\sum_{j=1}^{p}\left(\left|z_{j}\right|-N_{j}\right)=|Z|-(N-1)>0$ so there exists a $k$ such that $N_{k}<\left|z_{k}\right|$. We then have, for all $d \geq 1$,

$$
\begin{aligned}
& \liminf _{t \rightarrow+\infty}\left(\Sigma^{N}(R(t), Z)-\lambda_{d}^{N}(R(t), Z)\right) \\
& \geq \sum_{j=1}^{p} E^{N_{j}}\left(r_{j}, z_{j}\right)-\left(\lambda_{d}^{N_{k}+1}\left(r_{k}, z_{k}\right)+\sum_{j=1, j \neq k}^{p} E^{N_{j}}\left(r_{j}, z_{j}\right)\right) \\
& \quad=E^{N_{k}}\left(r_{k}, z_{k}\right)-\lambda_{d}^{N_{k}+1}\left(r_{k}, z_{k}\right)=\Sigma^{N_{k}+1}\left(r_{k}, z_{k}\right)-\lambda_{d}^{N_{k}+1}\left(r_{k}, z_{k}\right)
\end{aligned}
$$

and

$$
\begin{aligned}
\inf _{r_{i} \in \omega_{i}} \liminf _{t \rightarrow+\infty}\left(\Sigma^{N}(R(t), Z)-\lambda_{d}^{N}(R(t), Z)\right) \\
\geq \inf _{\substack{j=1, \ldots, p \\
n \leq\left|z_{j}\right|}} \inf _{\substack{j \in \omega_{j} \\
\text { na }}}\left(\Sigma^{n}\left(r_{j}, z_{j}\right)-\lambda_{d}^{n}\left(r_{j}, z_{j}\right)\right)>0
\end{aligned}
$$

by lemma 5 . The uniform exponential decay 4) is then a consequence of lemmas 3 and 4.

We now prove the inequality

$$
\liminf _{t \rightarrow+\infty} E^{N}(R(t), Z) \geq \min \left\{\sum_{j=1}^{p} E^{N_{j}}\left(r_{j}, z_{j}\right), N_{1}+\ldots+N_{p}=N\right\}
$$

by using a variant of classical $N$-body geometric methods for Schrödinger operators $[30,29,19]$, which is used in [10].

Let $t_{n} \rightarrow+\infty$ be such that $\lim _{n} E^{N}\left(R\left(t_{n}\right), Z\right)=\liminf _{t} E^{N}(R(t), Z)$, and $\Psi_{n}$ an associated sequence of ground states, with densities $\rho_{\Psi_{n}}$ and $t_{\Psi_{n}}$. We denote $R^{n}=R\left(t_{n}\right)$ and $X_{j}^{n}=X_{j}\left(t_{n}\right)$. Due to the uniform exponential decay, one has

$$
\lim _{n \rightarrow+\infty} \int_{\mathcal{U}\left(t_{n} / 3\right)} \rho_{\Psi_{n}}=\lim _{n \rightarrow+\infty} \int_{\mathcal{U}\left(t_{n} / 3\right)} t_{\Psi_{n}}=0 .
$$


Let $\xi_{n} \in \mathcal{C}^{\infty}\left(\mathbb{R}^{3},[0 ; 1]\right)$ be a cutoff function such that $\xi_{n} \equiv 0$ on $\mathcal{U}\left(t_{n} / 3\right)$, $\xi_{n} \equiv 1$ on $\mathbb{R}^{3} \backslash \mathcal{U}\left(t_{n} / 3-1\right)$, and $\left\|\nabla \xi_{n}\right\|_{\infty} \leq 1,\left\|\Delta \xi_{n}\right\|_{\infty} \leq 2$. We then introduce $\chi_{n}\left(x_{1}, \ldots, x_{N}\right)=\prod_{i=1}^{N} \xi_{n}\left(x_{i}\right)$ and $\tilde{\Psi}_{n}=\chi_{n} \Psi_{n}$. Using (12), it is then easy to see that $\left\|\Psi_{n}-\tilde{\Psi}_{n}\right\|_{H^{1}} \rightarrow 0$, and

$$
\begin{aligned}
& H^{N}\left(R^{n}, Z\right) \cdot \tilde{\Psi}_{n}-\lambda_{1}^{N}\left(R^{n}, Z\right) \cdot \tilde{\Psi}_{n}=-\sum_{i=1}^{N}\left(2 \nabla_{x_{i}} \chi_{n} \cdot \nabla_{x_{i}} \Psi_{n}+\Psi_{n} \Delta_{x_{i}} \chi_{n}\right) \\
& \rightarrow 0 \text { in } L^{2}\left(\mathbb{R}^{3 N}\right) \\
& \mathcal{E}^{N}\left(R^{n}, \tilde{\Psi}_{n}\right)-\lambda_{1}^{N}\left(R^{n}, \Psi_{n}\right)\left\|\tilde{\Psi}_{n}\right\|_{L^{2}\left(\mathbb{R}^{3 N}\right)}=\sum_{i=1}^{N} \int\left|\Psi_{n}\right|^{2}\left|\nabla_{x_{i}} \chi_{n}\right|^{2} \rightarrow 0
\end{aligned}
$$

Now, we may write $\xi_{n}=\sum_{j=1}^{p} \xi_{n}^{j}$ where $\operatorname{Supp}\left(\xi_{n}^{j}\right) \subset B\left(X_{j}, t_{n} / 3\right)$, and

$$
\tilde{\Psi}_{n}=\sum_{1 \leq k_{j} \leq p} \xi_{n}^{k_{1}}\left(x_{1}\right) \cdots \xi_{n}^{k_{N}}\left(x_{N}\right) \Psi_{n}:=\sum_{1 \leq k_{j} \leq p} \Psi_{n}^{k_{1}, \ldots, k_{N}} .
$$

Since the $\Psi_{n}^{k_{1}, \ldots, k_{N}}$ have disjoint supports,

$$
\begin{gathered}
\left|\tilde{\Psi}_{n}\right|^{2}=\sum_{1 \leq k_{j} \leq p}\left|\Psi_{n}^{k_{1}, \ldots, k_{N}}\right|^{2}, \quad \mathcal{E}^{N}\left(R^{n}, \tilde{\Psi}_{n}\right)=\sum_{1 \leq k_{j} \leq p} \mathcal{E}^{N}\left(R_{n}, \Psi_{n}^{k_{1}, \ldots, k_{N}}\right), \\
\left(H^{N}\left(R^{n}, Z\right)-\lambda_{1}^{N}\left(R^{n}, Z\right)\right) \cdot \Psi_{n}^{k_{1}, \ldots, k_{N}} \rightarrow 0
\end{gathered}
$$

in $L^{2}\left(\mathbb{R}^{3 N}\right)$ for all $k_{1}, \ldots k_{N}$. To end the proof of Theorem 2 , it suffices to bound $\mathcal{E}^{N}\left(R^{n}, \tilde{\Psi}_{n}\right)$ from below by the appropriate constant.. We now fix $k_{1}, \ldots, k_{N}$ and introduce $C_{j}=\left\{i, k_{i}=j\right\}, N_{j}=\left|C_{j}\right|$. Remark that $\Psi_{n}^{k_{1}, \ldots, k_{N}}$ is antisymmetric in $\left(x_{i}\right)_{i \in C_{j}}$ for all $j=1, \ldots, p$. Then

$$
\begin{aligned}
\mathcal{E}^{N}\left(R^{n}, \Psi_{n}^{k_{1}, \ldots, k_{N}}\right)= & \sum_{j=1}^{p}\left(\sum_{i \in C_{j}} \int \frac{1}{2}\left|\nabla_{x_{i}} \Psi_{n}^{k_{1}, \ldots, k_{N}}\right|^{2}-\sum_{i \in C_{j}} \int V_{\tilde{r}_{j}}\left(x_{i}\right)\left|\Psi_{n}^{k_{1}, \ldots, k_{N}}\right|^{2}\right. \\
& \left.+\sum_{k, l \in C_{j}} \int \frac{\left|\Psi_{n}^{k_{1}, \ldots, k_{N}}\right|^{2}}{\left|x_{k}-x_{l}\right|}+E^{0}\left(r_{j}, z_{j}\right)\right)+e_{n}
\end{aligned}
$$

where

$$
e_{n}=\sum_{1 \leq j \neq j^{\prime} \leq p}\left(\sum_{i \in C_{j}} \int V_{\tilde{r}_{j^{\prime}}}\left(x_{i}\right)\left|\Psi_{n}^{k_{1}, \ldots, k_{N}}\right|^{2}+\sum_{i \in C_{j}} \sum_{i^{\prime} \in C_{j^{\prime}}} \int \frac{\left|\Psi_{n}^{k_{1}, \ldots, k_{N}}\right|^{2}}{\left|x_{i}-x_{i^{\prime}}\right|}\right)+e_{n}^{\prime},
$$

$e_{n}^{\prime}$ being the interaction energy between nuclei in different molecules, which easily tends to 0 as $n \rightarrow+\infty$. Now

$$
\left|e_{n}\right| \leq \sum_{1 \leq j \neq j^{\prime} \leq p}\left(\sum_{i \in C_{j}} \int \frac{3\left|z_{j}\right|}{t_{n}}\left|\Psi_{n}^{k_{1}, \ldots, k_{N}}\right|^{2}+3 \sum_{i \in C_{j}} \sum_{i^{\prime} \in C_{j^{\prime}}} \int \frac{\left|\Psi_{n}^{k_{1}, \ldots, k_{N}}\right|^{2}}{t_{n}}\right)+e_{n}^{\prime} \rightarrow 0
$$


as $n \rightarrow+\infty$. Finally, since $\Psi_{n}^{k_{1}, \ldots, k_{N}}$ is antisymmetric in $\left(x_{i}\right)_{i \in C_{j}}$ for all $j=$ $1, \ldots, p$ and thanks to the translation invariance of the Hamiltonian,

$$
\mathcal{E}^{N}\left(R^{n}, \Psi_{n}^{k_{1}, \ldots, k_{N}}\right) \geq\left(\sum_{j=1}^{p} E^{N_{j}}\left(r_{j}, z_{j}\right)\right)\left\|\Psi_{n}^{k_{1}, \ldots, k_{N}}\right\|_{L^{2}}^{2}+e_{n} .
$$

Passing to the limit, we obtain

$$
\lim _{n \rightarrow+\infty} \mathcal{E}^{N}\left(R^{n}, \tilde{\Psi}_{n}\right) \geq \min \left\{\sum_{j=1}^{p} E^{N_{j}}\left(r_{j}, z_{j}\right), N_{1}+\cdots N_{p}=N\right\}
$$

which ends the proof of Theorem 2 .

\subsubsection{Proof of Theorem 3}

The proof uses exactly the same $N$-body geometric method as the end of the proof of Theorem 2, but with $\lambda_{1}^{N}\left(R^{n}, Z\right)$ replaced by $\lambda_{d}^{N}\left(R^{n}, Z\right)$. If we suppose that $\lim _{n \rightarrow+\infty} \lambda_{d}^{N}\left(R^{n}, Z\right)=c$, then passing to the limit and using Theorem 2

$$
c \leq \limsup _{n \rightarrow+\infty} \lambda_{d}^{N}\left(R^{n}, Z\right) \leq \Lambda_{d}^{N}(r, z) .
$$

We have

$$
\left(H^{N}\left(R^{n}, Z\right)-c\right) \cdot \Psi_{n}^{k_{1}, \ldots, k_{N}} \rightarrow 0
$$

in $L^{2}\left(\mathbb{R}^{3 N}\right)$ for all $k_{1}, \ldots k_{N}$. Since all the interaction terms tend to 0 (see the proof of Theorem 2), we obtain

$$
\left(\sum_{j=1}^{p} H^{N_{j}}\left(X_{j}^{n}+r_{j}^{n}, z_{j}\right)_{C_{j}}-c\right) \cdot \Psi_{n}^{k_{1}, \ldots, k_{N}} \rightarrow 0
$$

where the Hamiltonian $H^{N_{j}}\left(X_{j}^{n}+r_{j}^{n}, z_{j}\right)_{C_{j}}$ acts on the variables $\left(x_{i}\right)_{i \in C_{j}}$. Due to the translation invariance, we obtain

$$
\left(\sum_{j=1}^{p} H^{N_{j}}\left(r_{j}^{n}, z_{j}\right)_{C_{j}}-c\right) \cdot \tilde{\Psi}_{n}^{k_{1}, \ldots, k_{N}} \rightarrow 0
$$

where $\tilde{\Psi}_{n}^{k_{1}, \ldots, k_{N}}\left(x_{1}, \ldots, x_{N}\right)=\Psi_{n}^{k_{1}, \ldots, k_{N}}\left(X_{k_{i}}^{n}+x_{i}\right)$. But due to the exponential decay of $\Psi_{n}, \tilde{\Psi}_{n}^{k_{1}, \ldots, k_{N}}$ is precompact in $H^{1}\left(\mathbb{R}^{3 N}\right)$ and converges up to a subsequence to some $\tilde{\Psi}^{k_{1}, \ldots, k_{N}}$ such that

$$
\left(\sum_{j=1}^{p} H^{N_{j}}\left(r_{j}, z_{j}\right)_{C_{j}}\right) \tilde{\Psi}^{k_{1}, \ldots, k_{N}}=c \cdot \tilde{\Psi}^{k_{1}, \ldots, k_{N}} .
$$

We thus have either $c$ is an eigenvalue of $\left(\sum_{j=1}^{p} H^{N_{j}}\left(r_{j}, z_{j}\right)_{C_{j}}\right)$ on the tensor product $\bigotimes_{j=1}^{p} L_{a}^{2}\left(\mathbb{R}^{3 N_{j}}\right)_{C_{j}}$ (with an obvious notation), or $\tilde{\Psi}^{k_{1}, \ldots, k_{N}}=0$. 
Lemma 7. We have $\sigma\left(\sum_{j=1}^{p} H^{N_{j}}\left(r_{j}, z_{j}\right)_{C_{j}}\right)=\sum_{j=1}^{p} \sigma\left(H^{N_{j}}\left(r_{j}, z_{j}\right)_{C_{j}}\right)$ so that $\sigma_{\text {ess }}\left(\sum_{j=1}^{p} H^{N_{j}}\left(r_{j}, z_{j}\right)_{C_{j}}\right)=[\Sigma ;+\infty)$ with

$$
\begin{aligned}
\Sigma & =\min \left\{\sum_{j \neq j_{0}} E^{N_{j}}\left(r_{j}, z_{j}\right)+\Sigma^{N_{j_{0}}}\left(r_{j_{0}}, z_{j_{0}}\right), 1 \leq j_{0} \leq p\right\} \\
& >\Lambda_{d}^{N}(r, z)
\end{aligned}
$$

for all $d \geq 1$.

Proof of Lemma 7 - The fact that the spectrum of $\sum_{j=1}^{p} H^{N_{j}}\left(r_{j}, z_{j}\right)_{C_{j}}$ is the sum $\sum_{j=1}^{p} \sigma\left(H^{N_{j}}\left(r_{j}, z_{j}\right)_{C_{j}}\right)$ is standard (see for instance [27], Theorem VIII-33). Suppose now that $\Sigma=\sum_{j \neq j_{0}} E^{N_{j}}\left(r_{j}, z_{j}\right)+\Sigma^{N_{j_{0}}}\left(r_{j_{0}}, z_{j_{0}}\right)$ for some $1 \leq j_{0} \leq p$. If $\lambda_{d}^{N_{j_{0}}}\left(r_{j_{0}}, z_{j_{0}}\right)<\Sigma^{N_{j_{0}}}\left(r_{j_{0}}, z_{j_{0}}\right)$ then obviously $\Sigma>\Lambda_{d}^{N}(r, z)$. If $\lambda_{d}^{N_{j_{0}}}\left(r_{j_{0}}, z_{j_{0}}\right)=\Sigma^{N_{j_{0}}}\left(r_{j_{0}}, z_{j_{0}}\right)$ then $\Sigma>\Lambda_{d}^{N}(r, z)$ by lemma 6 .

As a consequence, if $c$ is an eigenvalue of $\left(\sum_{j=1}^{p} H^{N_{j}}\left(r_{j}, z_{j}\right)_{C_{j}}\right)$, it is necessary below its essential spectrum. It is then easy to see that this implies

$$
\tilde{\Psi}^{k_{1}, \ldots, k_{N}} \in \bigotimes_{j=1}^{p} \operatorname{ker}\left(H^{N_{j}}\left(r_{j}, z_{j}\right)-\lambda_{\delta_{j}}^{N_{j}}\left(r_{j}, z_{j}\right)\right)_{C_{j}}
$$

for some $\lambda_{\delta_{j}}^{N_{j}}\left(r_{j}, z_{j}\right)<\Sigma^{N_{j}}\left(r_{j}, z_{j}\right)$.

Now, we have $\left\|\Psi^{n}-\tau_{n} \cdot \Psi\right\|_{H_{a}^{1}\left(\mathbb{R}^{3 N}\right)} \rightarrow 0$ where $\Psi=\sum_{k_{1}, \ldots, k_{N}} \tilde{\Psi}^{k_{1}, \ldots, k_{N}}$.

\subsection{Proof of Theorem 4}

We may suppose $c>\max \left(\mathcal{E}^{N}(R, \Psi), \mathcal{E}^{N}\left(R^{\prime}, \Psi^{\prime}\right)\right)$.

Let us first prove the equality $c=c^{\prime}$. Indeed, $c^{\prime} \leq c$ is obvious. Let be $r_{n} \in \mathcal{R}$ a sequence such that $m_{n}:=\max _{t \in[0 ; 1]} E^{N}\left(r_{n}(t), Z\right) \rightarrow c^{\prime}$ as $n \rightarrow+\infty$. For each $n \in \mathbb{N}$, we define

$$
\begin{gathered}
c_{n}=\inf _{\psi \in \Gamma_{\Psi}} \max _{t \in[0 ; 1]} \mathcal{E}^{N}\left(r_{n}(t), \psi(t)\right) \\
\Gamma_{\Psi}=\left\{\psi \in \mathcal{C}^{0}\left([0 ; 1], S H_{a}^{1}\left(\mathbb{R}^{3 N}\right)\right), \psi(0)=\Psi, \psi(1)=\Psi^{\prime}\right\} .
\end{gathered}
$$

We may now apply the methods of [8] to obtain some sequences $t_{k} \in[0 ; 1]$ and $\left(\Psi_{k}\right)_{k \geq 1}$ such that

1. $\lim _{k \rightarrow+\infty} \mathcal{E}^{N}\left(r^{n}\left(t_{k}\right), \Psi_{k}\right)=c_{n}$

2. $H^{N}\left(r_{n}\left(t_{k}\right), Z\right) \cdot \Psi_{k}-\mathcal{E}^{N}\left(r_{n}\left(t_{k}\right), \Psi_{k}\right) \cdot \Psi_{k} \rightarrow 0$ in $L^{2}\left(\mathbb{R}^{3 N}\right)$,

3. $\mathcal{E}^{N}\left(r_{n}\left(t_{k}\right), \Psi_{k}\right) \leq \lambda_{1}^{N}\left(r_{n}\left(t_{k}\right), Z\right)+\epsilon_{k}, \quad \lim _{k \rightarrow+\infty} \epsilon_{k}=0$ 
$1,2)$ correspond to the classical fact that one can obtain min-maxing sequences that are almost critical. On the other hand, 3) is a consequence of the lessknown fact that one can obtain Palais-Smale sequences with Morse-type information related to the dimension of the homotopy-stable class used in the min-max method, which is 1 here (paths are deformations of $[0 ; 1]$ ). Since we are in $\mathbb{C}=\mathbb{R}^{2}$, eigenvectors always have an even Morse index and this is why $\lambda_{1}^{N}$ appears in 3) and not $\lambda_{2}^{N}$.

The fact that such a sequence $\left(\Psi_{k}\right)$ is precompact in $H_{a}^{1}\left(\mathbb{R}^{3 N}\right)$ is now a simple consequence of Theorem 1 - 4). Indeed, the compactness below the essential spectrum is nothing else but the Palais-Smale condition of $\mathcal{E}^{N}$ with Morse-type information introduced in [14]. We have the following general lemma, whose proof is postponed until the end of the proof of Theorem 4 .

Lemma 8. We assume that $Z=\left(Z_{1}, \ldots, Z_{M}\right)$ is such that $N \leq|Z|$. Let $\left(R^{n}, \Psi^{n}\right)$ be a sequence in $\Omega \times S H_{a}^{1}\left(\mathbb{R}^{3 N}\right)$ such that

1. $R^{n} \rightarrow R \in \Omega$

2. $\lim _{n \rightarrow+\infty} \mathcal{E}^{N}\left(R^{n}, \Psi^{n}\right)=c$,

3. $H^{N}\left(R^{n}, Z\right) \cdot \Psi^{n}-\mathcal{E}^{N}\left(R^{n}, \Psi^{n}\right) \cdot \Psi^{n} \rightarrow 0$ in $L^{2}\left(\mathbb{R}^{3 N}\right)$,

4. there exist $d_{0} \geq 1$ and $\epsilon_{n} \rightarrow 0$ such that $\mathcal{E}^{N}\left(R^{n}, \Psi^{n}\right) \leq \lambda_{d_{0}}^{N}\left(R^{n}, Z\right)+\epsilon_{n}$.

Then $\left(\Psi^{n}\right)$ is precompact in $H_{a}^{1}\left(\mathbb{R}^{3 N}\right)$ and converges, up to a subsequence, to an eigenfunction $\Psi$ of $H^{N}(R, Z)$ associated to $\lambda_{d}^{N}(R, Z)$ with $d \leq d_{0}$.

Applying this result, we obtain, by passing to the limit as $k \rightarrow+\infty$,

$$
c \leq c_{n}=\lambda_{1}^{N}\left(r_{n}\left(t_{n}\right), Z\right) \leq m_{n}
$$

for some $t_{n} \in[0 ; 1]$ and so $c=c^{\prime}$.

We now prove the alternative of the Theorem. We introduce

$$
\begin{gathered}
\mathcal{F}_{c}\left(R_{0}\right)=\left\{(R, \Psi) \in \Omega \times S H_{a}^{1}\left(\mathbb{R}^{3 N}\right), \sum_{i \neq j}\left|R_{i}-R_{j}\right| \leq R_{0}, \mathcal{E}^{N}(R, \Psi) \geq c\right\} \\
\Gamma(\alpha)=\left\{\gamma \in \Gamma, \max _{t \in[0 ; 1]} \mathcal{E}^{N}(\gamma(t)) \leq c+\alpha\right\} .
\end{gathered}
$$

We have the following alternative:

either there exist $R_{0}>0$ and $\alpha>0$ such that, $\mathcal{F}_{c}\left(R_{0}\right) \cap \gamma([0 ; 1]) \neq \emptyset$ for all $\gamma \in \Gamma(\alpha)$,

or for all $R_{0}>0$ there exists a min-maxing sequence $\gamma_{n} \in \Gamma$ such that $\mathcal{F}_{c}\left(R_{0}\right) \cap$ $\gamma_{n}([0 ; 1])=\emptyset$. 
First Case : there exist $R_{0}>0$ and $\alpha>0$ such that, $\mathcal{F}_{c}\left(R_{0}\right) \cap \gamma([0 ; 1]) \neq \emptyset$ for all $\gamma \in \Gamma(\alpha)$.

Since $(R, \Psi)$ and $\left(R^{\prime}, \Psi^{\prime}\right)$ do not belong to $\mathcal{F}_{c}\left(R_{0}\right)$, we may apply the methods of [14] $\left(\mathcal{F}_{c}\left(R_{0}\right)\right.$ is a set which is dual to the homotopy-stable class $\Gamma(\alpha)$ with boundary $\left.B=\left\{(R, \Psi),\left(R^{\prime}, \Psi^{\prime}\right)\right\}\right)$ to obtain a sequence $\left(R^{n}, \Psi^{n}\right) \in$ $\Omega \times S H_{a}^{1}\left(\mathbb{R}^{3 N}\right)$ such that

1. $\lim _{n \rightarrow+\infty} d\left(\left(R^{n}, \Psi^{n}\right), \mathcal{F}_{c}\left(R_{0}\right)\right)=0$,

2. $\lim _{n \rightarrow+\infty} \mathcal{E}^{N}\left(R^{n}, \Psi^{n}\right)=c$,

3. $\lim _{n \rightarrow+\infty} \nabla_{R} \mathcal{E}^{N}\left(R^{n}, \Psi^{n}\right)=0$,

4. $H^{N}\left(R^{n}, Z\right) \cdot \Psi^{n}-\mathcal{E}^{N}\left(R^{n}, \Psi^{n}\right) \cdot \Psi^{n} \rightarrow 0$ in $L^{2}\left(\mathbb{R}^{3 N}\right)$,

5. $\mathcal{E}^{N}\left(R^{n}, \Psi^{n}\right) \leq \lambda_{1}^{N}\left(R^{n}, Z\right)+\epsilon_{n}, \quad \lim _{n \rightarrow+\infty} \epsilon_{n}=0$

Remark that $2,3,4,5)$ correspond to the fact that one can obtain minmaxing sequences that are almost critical, and with Morse-type information. On the other hand, 1) is the consequence of the duality theory developed in $[14]$ that enables to locate the critical points.

Due to 1$), \sum_{i \neq j}\left|R_{i}^{n}-R_{j}^{n}\right|$ is bounded. Up to a translation, we may suppose $R^{n} \rightarrow R \in \Omega$ (since $\lambda_{1}^{N}\left(R^{n}, Z\right) \rightarrow+\infty$ when $d\left(R^{n}, \partial \Omega\right) \rightarrow 0$ due to the nuclei/nuclei repulsion). Now $\Psi^{n}$ converges up to a subsequence to a $\Psi$ in $H^{1}\left(\mathbb{R}^{3 N}\right)$ by lemma 8 .

Second Case : for all $R_{0}>0$ there exists a min-maxing sequence $\gamma_{n} \in \Gamma$ such that $\mathcal{F}_{c}\left(R_{0}\right) \cap \gamma_{n}([0 ; 1])=\emptyset$.

Let $\left(r_{n}\right)$ be a sequence in $\mathbb{R}$ such that $r_{n} \rightarrow+\infty$. For each $r_{n}$, there exists a $\gamma_{n}$ such that, for instance,

$$
c \leq \max _{t \in[0 ; 1]} \mathcal{E}^{N}\left(\gamma_{n}(t)\right) \leq c+\frac{1}{n}
$$

and $\mathcal{F}_{c}\left(r_{n}\right) \cap \gamma_{n}([0 ; 1])=\emptyset$. We now write $\gamma_{n}(t)=\left(R_{n}(t), \tilde{\Psi}_{n}(t)\right)$ and fix $n$. The set $K_{n}=\left\{t \in[0 ; 1], \sum\left|R_{i}^{n}(t)-R_{j}^{n}(t)\right| \leq r_{n}\right\}$ is a compact subset of $[0 ; 1]$ such that $\max \mathcal{E}^{N}\left(\gamma\left(K_{n}\right)\right)<c$. We now introduce

$$
\Gamma_{\Psi}=\left\{\psi \in \mathcal{C}^{0}\left([0 ; 1], S H_{a}^{1}\left(\mathbb{R}^{3 N}\right)\right), \psi_{\mid K_{n}} \equiv \tilde{\Psi}_{\mid K_{n}}^{n}\right\}
$$

which is an homotopy-stable class of dimension 1 with boundary $\tilde{\Psi}^{n}\left(K_{n}\right)$, and

$$
c_{n}=\inf _{\psi \in \Gamma_{\Psi}} \max _{t \in[0 ; 1]} \mathcal{E}^{N}\left(R_{n}(t), \psi(t)\right)
$$

so that

$$
c \leq c_{n} \leq c+\frac{1}{n}
$$

Applying the methods of [14], we may find a sequence $t_{k} \in[0 ; 1] \backslash K_{n}$ and $\Psi_{n}^{k} \in S H_{a}^{1}\left(\mathbb{R}^{3 N}\right)$ such that $t_{k} \rightarrow \bar{t}$ and 
1. $\sum_{i \neq j}\left|R_{n, i}\left(t_{k}\right)-R_{n, j}\left(t_{k}\right)\right| \geq r_{n}$

2. $\lim _{k \rightarrow+\infty} \mathcal{E}^{N}\left(R_{n}\left(t_{k}\right), \Psi_{n}^{k}\right)=c_{n}$

3. $H^{N}\left(R_{n}\left(t_{k}\right), Z\right) \cdot \Psi_{n}^{k}-\mathcal{E}^{N}\left(R_{n}\left(t_{k}\right), \Psi_{n}^{k}\right) \cdot \Psi_{n}^{k} \rightarrow 0 \quad$ in $L^{2}\left(\mathbb{R}^{3 N}\right)$

4. $\mathcal{E}^{N}\left(R_{n}\left(t_{k}\right), \Psi_{n}^{k}\right) \leq \lambda_{1}^{N}\left(R_{n}\left(t_{k}\right), Z\right)+\alpha_{k}$ with $\lim _{k \rightarrow+\infty} \alpha_{k}=0$

By lemma $8,\left(\Psi_{n}^{k}\right)_{k \in \mathbb{N}}$ is precompact in $H_{a}^{1}\left(\mathbb{R}^{3 N}\right)$ and converges, up to a subsequence, to some $\Psi^{n}$ such that

1. $\sum_{i \neq j}\left|R_{i}^{n}-R_{j}^{n}\right| \geq r_{n}$

2. $\lim _{n \rightarrow+\infty} \mathcal{E}^{N}\left(R^{n}, \Psi^{n}\right)=c$

3. $H^{N}\left(R^{n}, Z\right) \cdot \Psi^{n}=\lambda_{1}^{N}\left(R^{n}, Z\right) \cdot \Psi^{n}$

where $R^{n}:=R_{n}(\bar{t})$.

Since $\sum_{i \neq j}\left|R_{i}^{n}-R_{j}^{n}\right| \rightarrow+\infty$, there exists a $2 \leq p \leq M$, some $X_{j}^{n} \in \mathbb{R}^{3}$ with $j=1, \ldots, p$ and a $R_{0}>0$ such that (changing the indices if necessary and up to a subsequence) $R^{n}=\left(X_{1}^{n}+r_{1}^{n}, \ldots, X_{p}^{n}+r_{p}^{n}\right),\left\|r_{j}^{n}\right\| \leq R_{0}, Z=\left(z_{1}, \ldots, z_{p}\right)$, and $\lim _{n \rightarrow+\infty}\left|X_{i}^{n}-X_{j}^{n}\right|=+\infty, r_{j}^{n} \rightarrow r_{j}$.

Passing to the limit, we obtain, by Theorem 2

$$
c=\lim _{n \rightarrow+\infty} \lambda_{1}^{N}\left(R^{n}, Z\right)=\Lambda_{1}^{N}(r, z) .
$$

We now simply apply Theorem 3 to obtain the convergence to a critical point at infinity of energy $c$, as defined in the corresponding section.

Let us now prove Lemma 8.

Proof of Lemma 8 - Let $m$ be an integer such that $\lambda_{m}^{N}(R, Z)>\lambda_{m-1}^{N}(R, Z)=$ $\lambda_{d_{0}}^{N}(R, Z)$. Due to the fact that $\lambda_{d}^{N}\left(R^{n}, Z\right) \rightarrow \lambda_{d}^{N}(R, Z)$ as $n \rightarrow+\infty$, we have $\lambda_{m}^{N}\left(R^{n}, Z\right)>\lambda_{d_{0}}^{N}(R, Z) \geq c$ for $n$ large enough.

Let be $V_{n}=\bigoplus_{i=1}^{m-1} \operatorname{ker}\left(H\left(R^{n}, Z\right)-\lambda_{i}^{N}\left(R^{n}, Z\right)\right)$, and $\left(\psi_{1}^{n}, \ldots, \psi_{m-1}^{n}\right)$ an orthonormal basis of $V^{n}, \psi_{i}^{n}$ being eigenfunctions of $H^{N}\left(R^{n}, Z\right)$ (Theorem 1 - 4)). Due for instance to the uniform exponential decay of Theorem 2, one easily sees that each $\psi_{i}^{n}$ is precompact and converges up to a subsequence in $H_{a}^{1}\left(\mathbb{R}^{3 N}\right)$ to a $\psi_{i}$, with $\operatorname{span}\left(\psi_{i}\right)=\bigoplus_{i=1}^{m-1} \operatorname{ker}\left(H(R, Z)-\lambda_{i}^{N}(R, Z)\right)$.

Now we can write $\Psi^{n}=\Psi_{V^{n}}+\Psi_{\left(V^{n}\right) \perp}$ with an obvious definition. Since $\mathcal{E}^{N}\left(R^{n}, \Psi^{n}\right)$ is bounded, it is a classical fact that $\left(\Psi^{n}\right)$ is bounded in $H_{a}^{1}\left(\mathbb{R}^{3 N}\right)$ and so, up to a subsequence, $\Psi^{n} \rightarrow \Psi$ weakly in $H_{a}^{1}\left(\mathbb{R}^{3 N}\right)$. Since $\operatorname{dim}\left(V_{n}\right)=$ $m-1,\left(\Psi_{V_{n}}\right)$ is precompact in $H^{1}\left(\mathbb{R}^{3 N}\right)$ and converges to a $\Psi_{V} \in V$. By difference, $\Psi_{\left(V^{n}\right) \perp} \rightarrow \Psi_{(V) \perp}$ weakly in $H^{1}\left(\mathbb{R}^{3 N}\right)$.

Since $\lim _{n \rightarrow+\infty} \mathcal{E}^{N}\left(R^{n}, \Psi^{n}\right)=c, H^{N}\left(R^{n}, Z\right) \cdot \Psi^{n}-c \cdot \Psi^{n} \rightarrow 0$ in $L^{2}\left(\mathbb{R}^{3 N}\right)$, so we obtain

$$
\left(H^{N}\left(R^{n}, Z\right)-c\right) \cdot \Psi_{V_{n}}+\left(H^{N}\left(R^{n}, Z\right)-c\right) \cdot \Psi_{\left(V_{n}\right)^{\perp}} \rightarrow 0
$$


which implies $\left(H^{N}\left(R^{n}, Z\right)-c\right) \cdot \Psi_{V_{n}} \rightarrow 0$ and $\left(H^{N}\left(R^{n}, Z\right)-c\right) \cdot \Psi_{\left(V_{n}\right)^{\perp}} \rightarrow 0$ in $L^{2}\left(\mathbb{R}^{3 N}\right)$. Finally,

$$
\mathcal{E}^{N}\left(R^{n}, \Psi_{\left(V_{n}\right)^{\perp}}\right)-c\left\|\Psi_{\left(V_{n}\right)^{\perp}}\right\|_{L^{2}\left(\mathbb{R}^{3 N}\right)}^{2} \rightarrow 0 .
$$

Because $\min \mathcal{E}^{N}\left(R^{n}, S\left(V_{n}\right)^{\perp}\right)=\lambda_{m}^{N}\left(R^{n}, Z\right)>\lambda_{d_{0}}^{N}\left(R^{n}, Z\right) \geq c$, this implies $\left\|\Psi_{\left(V_{n}\right)^{\perp}}\right\|_{L^{2}} \rightarrow 0$ and then $\left\|\Psi_{\left(V_{n}\right)^{\perp}}\right\|_{H^{1}} \rightarrow 0$. Thus $\Psi^{n}$ converges in $H^{1}\left(\mathbb{R}^{3 N}\right)$ to a $\Psi=\Psi_{V}$ which is an eigenfunction of $H^{N}(R, Z)$ that belongs to $V$.

\subsection{The case of two interacting molecules}

\subsubsection{Proof of Lemma 2}

We shall use the following lemma:

Lemma 9 (Multipole expansion). There exists a constant $C$ such that, for all $R$ and $h \in \mathbb{R}^{3}$ with $R+h \neq 0$,

$$
\left|\frac{1}{|R+h|}-\left(\frac{1}{|R|}-\frac{e_{R} \cdot h}{|R|^{2}}+\frac{3\left(e_{R} \cdot h\right)^{2}-|h|^{2}}{2|R|^{3}}\right)\right| \leq \frac{C|h|^{3}}{|R|^{3}|R+h|}
$$

with $e_{R}=R /|R|$.

Proof of Lemma 9 - It suffices to show

$$
\left|\frac{1}{\sqrt{1-2 x t+t^{2}}}-\left(1+x t+\frac{t^{2}}{2}\left(3 x^{2}-1\right)\right)\right| \leq \frac{C|t|^{3}}{\sqrt{1-2 x t+t^{2}}}
$$

for all $t \in \mathbb{R}$ and $x \in[-1 ; 1]$ (take $x=-\left(e_{R} \cdot h\right) /|h|$ and $\left.t=|h| /|R|\right)$ ). We thus introduce $f(x, t)=1-\sqrt{1-2 x t+t^{2}}\left(1+x t+\frac{t^{2}}{2}\left(3 x^{2}-1\right)\right)$. One easily computes $\frac{\partial f}{\partial x}(x, t)=\frac{3 t^{3}\left(5 x^{2}-2 x t-1\right)}{2 \sqrt{1-2 x t+t^{2}}}$, so that

$$
\max _{x \in[-1 ; 1]}|f(x, t)| \leq \max \left\{f_{1}(t), f_{2}(t), f_{3}(t), f_{4}(t)\right\}
$$

where $f_{1}(t)=\left|f\left(x_{1}(t), t\right)\right| \mathbb{1}_{-1 \leq x_{1} \leq 1}(t), f_{2}(t)=\left|f\left(x_{2}(t), t\right)\right| \mathbb{1}_{-1 \leq x_{2} \leq 1}(t), f_{3}(t)=$ $|f(-1, t)|, f_{4}(t)=|f(1, t)|, x_{1}(t)=\frac{t-\sqrt{t^{2}+5}}{5}$ and $x_{2}(t)=\frac{t+\sqrt{t^{2}+5}}{5}$. It is now easy to conclude that $|f(x, t)| \leq C|t|^{3}$ for some constant $C>0$.

We are now able to prove Lemma 2 .

Proof of Lemma 2 - Let $\xi_{\alpha} \in \mathcal{C}^{\infty}\left(\mathbb{R}^{3},[0 ; 1]\right)$ be a cut-off function such that $\xi_{\alpha} \equiv 0$ on $\mathbb{R}^{3} \backslash B(O, \alpha / 3), \xi_{\alpha} \equiv 1$ on $B(O, \alpha / 3-1),\left\|\nabla \xi_{\alpha}\right\|_{\infty} \leq 1,\left\|\Delta \xi_{\alpha}\right\|_{\infty} \leq 2$. We introduce $\tilde{\psi}_{j}^{\alpha}\left(x_{1}, \ldots, x_{N_{j}}\right)=\prod_{k=1}^{N_{j}} \xi_{\alpha}\left(x_{k}\right) \psi_{j}\left(x_{1}, \ldots, x_{N_{j}}\right), \psi_{j}^{\alpha}=\tilde{\psi}_{j}^{\alpha} /\left\|\tilde{\psi}_{j}^{\alpha}\right\|_{L^{2}}$ and $\tilde{\Psi}\left(\alpha, u, u^{\prime}\right)=\left(u \cdot \psi_{1}^{\alpha}\right) \wedge\left(\tau_{\alpha \vec{v}} \cdot u^{\prime} \cdot \psi_{2}^{\alpha}\right)$. Due to the exponential decay of Theorem 2, one has

$$
\begin{gathered}
\left|\mathcal{E}^{N}\left(R\left(\alpha, u, u^{\prime}\right), \tilde{\Psi}\left(\alpha, u, u^{\prime}\right)\right)-\mathcal{E}^{N}\left(R\left(\alpha, u, u^{\prime}\right), \Psi\left(\alpha, u, u^{\prime}\right)\right)\right| \leq C e^{-a \alpha} \\
\left|E^{N_{1}}(r, z)-\mathcal{E}^{N_{1}}\left(r, \psi_{1}^{\alpha}\right)\right|,\left|E^{N_{2}}\left(r^{\prime}, z^{\prime}\right)-\mathcal{E}^{N_{2}}\left(r^{\prime}, \psi_{2}^{\alpha}\right)\right| \leq C e^{-a \alpha}
\end{gathered}
$$


for some $C, a>0$. Let us recall that, by definition,

$$
\psi \wedge \psi^{\prime}\left(x_{1}, \ldots, x_{N}\right)=\sqrt{\frac{N_{1} ! N_{2} !}{N !}} \sum_{|C|=N_{1}} \sigma(C) \psi\left(x_{C}\right) \psi^{\prime}\left(x_{\bar{C}}\right)
$$

where $x_{C}=\left(x_{i_{1}}, \ldots, x_{i_{N_{1}}}\right)$ when $C=\left\{i_{1}<\cdots<i_{N_{1}}\right\}, \sigma(C)= \pm 1$. Applying this equality to $\tilde{\Psi}$, we obtain on the right functions with disjoint supports. We shall therefore only study the expansion of

$$
\mathcal{E}^{N}\left(R\left(\alpha, u, u^{\prime}\right),\left(u \cdot \psi_{1}^{\alpha}\right) \otimes\left(\tau_{\alpha \vec{v}} \cdot u^{\prime} \cdot \psi_{2}^{\alpha}\right)\right) .
$$

We have (using the notation $x^{1}=\left(x_{1}, \ldots, x_{N_{1}}\right)$ and $\left.x^{2}=\left(x_{N_{1}+1}, \ldots, x_{N}\right)\right)$

$$
\begin{aligned}
& \mathcal{E}^{N}\left(R\left(\alpha, u, u^{\prime}\right),\left(u \psi_{1}^{\alpha}\right) \otimes\left(\tau_{\alpha \vec{v}} u^{\prime} \psi_{2}^{\alpha}\right)\right)=\mathcal{E}^{N_{1}}\left(u r, u \psi_{1}^{\alpha}\right)+\mathcal{E}^{N_{2}}\left(\alpha \vec{v}+u^{\prime} r^{\prime}, \tau_{\alpha \vec{v}} u^{\prime} \psi_{2}^{\alpha}\right) \\
& +\sum_{i=1}^{N_{1}} \sum_{j=1}^{N_{2}} \int_{\mathbb{R}^{3 N}} \frac{\left|\psi_{1}^{\alpha}\left(x^{1}\right)\right|^{2}\left|\psi_{2}^{\alpha}\left(x^{2}\right)\right|^{2}}{\left|\alpha \vec{v}+u^{\prime} \cdot x_{j}^{2}-u \cdot x_{i}^{1}\right|} d x^{1} d x^{2}+\sum_{i=1}^{m} \sum_{j=1}^{m^{\prime}} \frac{z_{i} z_{j}^{\prime}}{\left|\alpha \vec{v}+u^{\prime} \cdot r_{j}^{\prime}-u \cdot r_{i}\right|} \\
& -\int_{\mathbb{R}^{3 N}}\left\{\sum_{i=1}^{m} \sum_{j=1}^{N_{2}} \frac{z_{i}\left|\psi_{1}^{\alpha}\left(x^{1}\right)\right|^{2}\left|\psi_{2}^{\alpha}\left(x^{2}\right)\right|^{2}}{\left|\alpha \vec{v}+u^{\prime} \cdot x_{j}^{1}-u \cdot r_{i}\right|}+\sum_{i=1}^{N_{1}} \sum_{j=1}^{m^{\prime}} \frac{z_{j}^{\prime}\left|\psi_{1}^{\alpha}\left(x^{1}\right)\right|^{2}\left|\psi_{2}^{\alpha}\left(x^{2}\right)\right|^{2}}{\left|\alpha \vec{v}+u^{\prime} \cdot r_{j}^{\prime}-u \cdot x_{i}^{1}\right|}\right\} d x^{1} d x^{2}
\end{aligned}
$$

so we obtain

$$
\begin{aligned}
& \mathcal{E}^{N}(\left.R\left(\alpha, u, u^{\prime}\right),\left(u \psi_{1}^{\alpha}\right) \otimes\left(\tau_{\alpha \vec{v}} u^{\prime} \psi_{2}^{\alpha}\right)\right)=\mathcal{E}^{N_{1}}\left(r, \psi_{1}^{\alpha}\right)+\mathcal{E}^{N_{2}}\left(r^{\prime}, \psi_{2}^{\alpha}\right) \\
& \quad+\iint_{\mathbb{R}^{6}} \frac{\rho_{1}^{\alpha}(x) \rho_{2}^{\alpha}(y)}{\left|\alpha \vec{v}+u^{\prime} \cdot y-u \cdot x\right|} d x d y+\sum_{i=1}^{m} \sum_{j=1}^{m^{\prime}} \frac{z_{i} z_{j}^{\prime}}{\left|\alpha \vec{v}+u^{\prime} \cdot r_{j}^{\prime}-u \cdot r_{i}\right|} \\
&-N_{1} \sum_{i=1}^{m} \int_{\mathbb{R}^{3}} \frac{z_{i} \rho_{2}^{\alpha}(y)}{\left|\alpha \vec{v}+u^{\prime} \cdot y-u \cdot r_{i}\right|} d y-N_{2} \sum_{j=1}^{m^{\prime}} \int_{\mathbb{R}^{3}} \frac{z_{j}^{\prime} \rho_{1}^{\alpha}(x)}{\left|\alpha \vec{v}+u^{\prime} \cdot r_{j}^{\prime}-u \cdot x\right|} d x
\end{aligned}
$$

where the $\rho_{k}^{\alpha}$ are the electronic densities associated to $\psi_{k}^{\alpha}$, and finally

$$
\begin{aligned}
\mathcal{E}^{N}\left(R\left(\alpha, u, u^{\prime}\right),\left(u \psi_{1}^{\alpha}\right) \otimes\left(\tau_{\alpha \vec{v}} u^{\prime} \psi_{2}^{\alpha}\right)\right)=\mathcal{E}^{N_{1}}\left(r, \psi_{1}^{\alpha}\right)+\mathcal{E}^{N_{2}}\left(r^{\prime}, \psi_{2}^{\alpha}\right) & \\
& +\iint_{\mathbb{R}^{6}} \frac{\tilde{\rho}_{1}^{\alpha}(x) \tilde{\rho}_{2}^{\alpha}(y)}{\left|\alpha \vec{v}+u^{\prime} \cdot y-u \cdot x\right|} d x d y
\end{aligned}
$$

where $\tilde{\rho}_{1}^{\alpha}(x)=\rho_{1}^{\alpha}(x)-\sum_{i=1}^{m} z_{i} \delta_{r_{i}}(x)$ and $\tilde{\rho}_{2}^{\alpha}(y)=\rho_{2}^{\alpha}(y)-\sum_{j=1}^{m^{\prime}} z_{i}^{\prime} \delta_{r_{i}^{\prime}}(y)$ are the total densities of charge associated to the distributions $\rho_{1}^{\alpha}$ and $\rho_{2}^{\alpha}$. Now, by lemma 9, we have

$$
\begin{array}{r}
\iint_{\mathbb{R}^{6}} \frac{\tilde{\rho}_{1}^{\alpha}(x) \tilde{\rho}_{2}^{\alpha}(y)}{\left|\alpha \vec{v}+u^{\prime} \cdot y-u \cdot x\right|} d x d y=\frac{q_{1} q_{2}}{\alpha}+q_{2} \frac{\left(u P_{1}^{\alpha}\right) \cdot \vec{v}}{\alpha^{2}}-q_{1} \frac{\left(u^{\prime} P_{2}^{\alpha}\right) \cdot \vec{v}}{\alpha^{2}} \\
-\frac{3\left(u P_{1}^{\alpha} \cdot \vec{v}\right)\left(u^{\prime} P_{2}^{\alpha} \cdot \vec{v}\right)-\left(u P_{1}^{\alpha}\right) \cdot\left(u^{\prime} P_{2}^{\alpha}\right)}{\alpha^{3}}+\frac{3\left(q_{2} u Q_{1} u^{T}+q_{1} u^{\prime} Q_{2} u^{\prime T}\right) v \cdot v}{2 \alpha^{3}} \\
+O\left(\frac{1}{\alpha^{3}} \iint_{\mathbb{R}^{6}} \frac{\left|u^{\prime} y-u x\right|^{3}\left|\tilde{\rho}_{1}^{\alpha}\right|(x)\left|\tilde{\rho}_{2}^{\alpha}\right|(y)}{\left|\alpha \vec{v}+u^{\prime} \cdot y-u \cdot x\right|} d x d y\right)
\end{array}
$$


But we have

$$
\iint_{\mathbb{R}^{6}} \frac{\left|u^{\prime} y-u x\right|^{3}\left|\tilde{\rho}_{1}^{\alpha}\right|(x)\left|\tilde{\rho}_{2}^{\alpha}\right|(y)}{\left|\alpha \vec{v}+u^{\prime} \cdot y-u \cdot x\right|} d x d y \leq \frac{3 C}{\alpha} \int_{\mathbb{R}^{3}}|x|^{3}\left(\left|\tilde{\rho}_{1}\right|+\left|\tilde{\rho}_{2}\right|\right)(x) d x .
$$

It suffices to notice that $\left|P_{k}^{\alpha}-P_{k}\right| \leq C e^{-a \alpha}$ and $\left|Q_{k}^{\alpha}-Q_{k}\right| \leq C e^{-a \alpha}$ for some $C, a>0$ to end the proof.

\subsubsection{Proof of Theorem 6}

Let us suppose that we are in the second case of Theorem 5, and that $c>$ $\max \left\{\mathcal{E}^{N}(M), \mathcal{E}^{N}\left(M^{\prime}\right)\right\}$. By the proof of Theorem 4, we obtain a sequence $\alpha_{n} \rightarrow+\infty$ and paths $\gamma_{n}$ such that

$$
\Lambda_{1}^{N} \leq c \leq \max _{t \in[0 ; 1]} \mathcal{E}^{N}\left(\gamma_{n}(t)\right) \leq c+\frac{1}{n}
$$

and $\mathcal{E}^{N}\left(\alpha(t), u(t), u^{\prime}(t), \Psi(t)\right)<c$ when $\alpha(t) \leq \alpha_{n}$. Let $t_{1}^{n}$ and $t_{2}^{n}$ be respectively the minimum and the maximum of $\left\{t, \alpha(t) \geq \alpha_{n}\right\}$. By the definition of $c$, we have $0<t_{1}^{n}<t_{2}^{n}<1$. For the sake of simplicity, we introduce $u_{j}^{n}=u\left(t_{j}^{n}\right)$, $u_{j}^{\prime n}=u^{\prime}\left(t_{j}^{n}\right)$, and $\Psi_{j}^{n}=\Psi\left(t_{j}^{n}\right)$.

The idea of the proof is now to connect $M_{1}^{n}=\left(\alpha_{n}, u_{1}^{n}, u_{1}^{\prime n}, \Psi_{1}^{n}\right)$ and $M_{2}^{n}=$ $\left(\alpha_{n}, u_{2}^{n}, u_{2}^{\prime n}, \Psi_{2}^{n}\right)$ by a path on which $\alpha$ is constant, with a maximum energy that is below $c$.

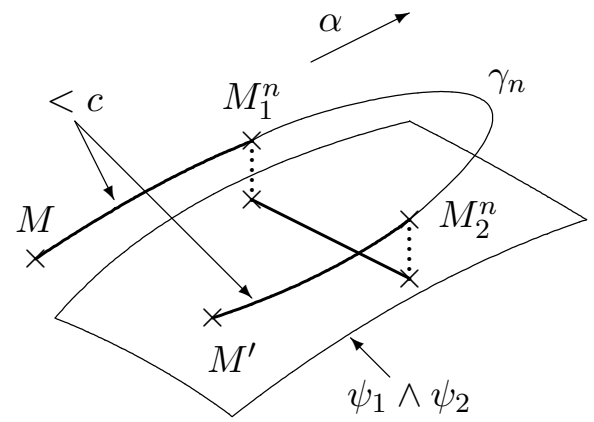

Figure 3: A schematic representation of the proof

We shall use the following lemma

Lemma 10. We assume that $\left(\alpha, u, u^{\prime}\right) \in(0 ;+\infty) \times\left(S_{3}(\mathbb{R})\right)^{2}$ is fixed, and we abbreviate $R=R\left(\alpha, u, u^{\prime}\right)$. Let $\Psi_{1}$ and $\Psi_{2}$ be two wavefunctions of $S H_{a}^{1}\left(\mathbb{R}^{3 N}\right)$. Then there exists a continuous path $\Psi:[0 ; 1] \rightarrow S H_{a}^{1}\left(\mathbb{R}^{3 N}\right)$ such that $\Psi(0)=$ $\Psi_{1}, \Psi(1)=\Psi_{2}$, and for all $t \in[0 ; 1]$

$$
\mathcal{E}^{N}(R, \Psi(t)) \leq \max \left\{\mathcal{E}^{N}\left(R, \Psi_{1}\right), \mathcal{E}^{N}\left(R, \Psi_{2}\right)\right\} .
$$


Proof of Lemma 10 - Let us denote by $V$ the finite-dimensional eigenspace associated to the first eigenvalue $\lambda_{1}^{N}(R, Z)$ of $H^{N}(R, Z)$, and let $P_{V}$ be the projection onto $V$. We may write $\Psi_{1}=t_{0} \Psi_{V}+\sqrt{1-t_{0}^{2}} \Psi_{V^{\perp}}$, where $t_{0} \in[0 ; 1]$, $\Psi_{V}=\frac{P_{V}\left(\Psi_{1}\right)}{\left\|P_{V}\left(\Psi_{1}\right)\right\|_{L^{2}}}$ if $P_{V}\left(\Psi_{1}\right) \neq 0$, and $\Psi_{V}$ is an arbitrary normalized function of $V$ if $P_{V}\left(\Psi_{1}\right)=0$ (then $t_{0}=0$ ). This enables to define a path connecting $\Psi_{1}$ to $\Psi_{V}$, on which the energy decreases, by varying $t \in\left[t_{0} ; 1\right]$.

We may find with the same method a path from $\Psi_{2}$ to some $\Psi_{V}^{\prime}$ in $V$, with a decreasing energy. It remains now to take an arbitrary path in the sphere of $V$ to connect $\Psi_{V}$ and $\Psi_{V}^{\prime}$ (the sphere of $V$ being pathwise-connected since we are in $\mathbb{C})$, on which $\mathcal{E}^{N}(R, \cdot)$ is constant.

Now, let $N_{1}$ and $N_{2}$ be such that

$$
E^{N_{1}}(r, z)+E^{N_{2}}\left(r^{\prime}, z^{\prime}\right)=\min \left\{E^{n_{1}}(r, z)+E^{n_{2}}\left(r^{\prime}, z^{\prime}\right), n_{1}+n_{2}=N\right\}:=\Lambda_{1}^{N}
$$

and $\left(N_{1}-|z|\right)\left(N_{2}-\left|z^{\prime}\right|\right) \neq 0$.

By lemma 6 , there exist $\psi_{1}$ and $\psi_{2}$, two ground states of respectively $H^{N_{1}}(r, z)$ and $H^{N_{2}}\left(r^{\prime}, z^{\prime}\right)$. We now introduce $\tilde{\Psi}_{k}^{n}=\left(u_{k}^{n} \cdot \psi_{1}\right) \wedge\left(\tau_{\alpha_{n} \vec{v}} \cdot u_{k}^{\prime n} \psi_{2}\right)$ for $k=1,2$. Since $S_{3}(\mathbb{R})$ is pathwise connected, one may find two paths $U_{n}, U_{n}^{\prime}:[0 ; 1] \rightarrow S \mathcal{O}_{3}(\mathbb{R})$ such that $U_{n}(0)=u_{1}^{n}, U_{n}(1)=u_{2}^{n}, U_{n}^{\prime}(0)=u_{1}^{\prime n}$ and $U_{n}^{\prime}(1)=u_{2}^{\prime n}$. This enables to connect $\tilde{M}_{1}^{n}:=\left(\alpha_{n}, u_{1}^{n}, u_{1}^{\prime n}, \tilde{\Psi}_{1}^{n}\right)$ and $\tilde{M}_{2}^{n}:=$ $\left(\alpha_{n}, u_{2}^{n}, u_{2}^{\prime n}, \tilde{\Psi}_{2}^{n}\right)$ by a path on which $\alpha \equiv \alpha_{n}$. Applying Lemma 10, we may connect $M_{1}^{n}$ to $\tilde{M}_{1}^{n}$, and $M_{2}^{n}$ to $\tilde{M}_{2}^{n}$. We finally obtain an admissible path $\tilde{\gamma}_{n}$ connecting $M$ and $M^{\prime}$, with a maximum that is attained "between" $\tilde{M}_{1}^{n}$ and $\tilde{M}_{2}^{n}$. It is now a consequence of Lemma 2 that

$\max _{t \in[0 ; 1]} \mathcal{E}^{N}\left(\tilde{\gamma}_{n}(t)\right)=E^{N_{1}}(r, z)+E^{N_{2}}\left(r^{\prime}, z^{\prime}\right)+\frac{\left(N_{1}-|z|\right)\left(N_{2}-\left|z^{\prime}\right|\right)}{\alpha_{n}}+O\left(\frac{1}{\left(\alpha_{n}\right)^{2}}\right)$.

Since $N_{1}+N_{2}=N=|z|+\left|z^{\prime}\right|$, we have $\left(N_{1}-|z|\right)\left(N_{2}-\left|z^{\prime}\right|\right)<0$ and so

$$
c \leq \max _{t \in[0 ; 1]} \mathcal{E}^{N}\left(\tilde{\gamma}_{n}(t)\right)<E^{N_{1}}(r, z)+E^{N_{2}}\left(r^{\prime}, z^{\prime}\right)
$$

for $n$ large enough, which is a contradiction.

\subsubsection{Proof of Theorem 7}

As in the previous proofs, we may suppose that $c>\max \left\{\mathcal{E}^{N}(M), \mathcal{E}^{N}\left(M^{\prime}\right)\right\}$, and that there exists a sequence $\alpha_{n} \rightarrow+\infty$ and paths $\gamma_{n}$ such that

$$
c \leq \max _{t \in[0 ; 1]} \mathcal{E}^{N}\left(\gamma_{n}(t)\right) \leq c+\frac{1}{n}
$$

and $\mathcal{E}^{N}\left(\alpha(t), u(t), u^{\prime}(t), \Psi(t)\right)<c$ when $\alpha(t) \leq \alpha_{n}$. We use the same definitions as above for $t_{1}^{n}, t_{2}^{n}, M_{k}^{n}=\left(u_{k}^{n}, u_{k}^{\prime n}, \Psi_{k}^{n}\right)$ for $k=1,2$. Applying Lemma 10 if necessary, we may also assume that $\Psi_{k}^{n}$ is a ground state of $H^{N}\left(R\left(\alpha_{n}, u_{k}^{n}, u_{k}^{\prime n}\right), Z\right)$.

Up to a subsequence, we may assume that $u_{k}^{n} \rightarrow u_{k}$ and $u_{k}^{\prime n} \rightarrow u_{k}^{\prime}$ as $n$ goes to $+\infty$ and that each $\left(\Psi_{k}^{n}\right)_{n}$ converges to some critical point at infinity of energy $c$ (by Theorem 3). But by (H1), (H3) (let us assume for instance that 
$E^{|z|}(r, z)$ is not degenerated), these points can be written $(u \psi) \wedge\left(u^{\prime} \psi^{\prime}\right)$ where $\psi$ is a fixed ground state of $H^{|z|}(r, z)$, and $\psi^{\prime} \in \operatorname{ker}\left(H^{\left|z^{\prime}\right|}\left(r^{\prime}, z^{\prime}\right)-E^{\left|z^{\prime}\right|}\left(r^{\prime}, z^{\prime}\right)\right)$, $\left\|\psi^{\prime}\right\|_{L^{2}}=1$. So finally, we may assume that each $\left(\Psi_{k}^{n}\right)_{n}$ converges to a critical point at infinity of the form $\left(u_{k} \psi\right) \wedge\left(u_{k}^{\prime} \psi_{k}^{\prime}\right)$.

Step 1: Rotating the molecules. As above, the idea is now to find a path from each $M_{k}^{n}$ to some tensor product, with a non-increasing energy. But we cannot apply the method used before since a tensor product may now have an energy which is greater than $c$ if the molecules have a 'bad' orientation. This is due to the fact that the first term in the expansion of the interaction energy will be the dipole/dipole term. Therefore, our first step will be to change the orientation of the molecules, so that the dipole/dipole interaction energy of a tensor product becomes negative.

Let $\xi_{1}^{n} \in \mathcal{C}^{\infty}\left(\mathbb{R}^{3},[0 ; 1]\right)$ be a cut-off function such that $\xi_{1}^{n} \equiv 0$ on $\mathbb{R}^{3} \backslash$ $B\left(O, \alpha_{n} / 3\right), \xi_{1}^{n} \equiv 1$ on $B\left(O, \alpha_{n} / 3-1\right),\left\|\nabla \xi_{1}^{n}\right\|_{\infty} \leq 1,\left\|\Delta \xi_{1}^{n}\right\|_{\infty} \leq 2$, and let us denote by $\xi_{2}^{n}=\tau_{\alpha_{n} \vec{v}} \xi_{1}^{n}$ the translation of $\xi_{1}^{n}$, and by $\xi_{3}^{n}:=1-\xi_{1}^{n}-\xi_{2}^{n}$. We now write as in the proof of Theorem 2

$\Psi_{k}^{n}=\prod_{l=1}^{N}\left(\xi_{1}^{n}+\xi_{2}^{n}+\xi_{3}^{n}\right)\left(x_{l}\right) \Psi_{k}^{n}=\sum_{1 \leq k_{l} \leq 3} \xi_{k_{1}}^{n}\left(x_{1}\right) \cdots \xi_{k_{N}}^{n}\left(x_{N}\right) \Psi_{k}^{n}:=\sum_{1 \leq k_{l} \leq 3}\left(\Psi_{k}^{n}\right)^{k_{1}, \ldots, k_{N}}$.

Since $k \in\{1,2\}$ and $n$ will be fixed during this step, we shall forget the subscripts and write $\Psi=\sum_{1 \leq k_{l} \leq 3} \Psi^{k_{1}, \ldots, k_{N}}$. We now introduce for all $v_{1}$, $v_{2} \in S \mathcal{O}_{3}(\mathbb{R})$,

$$
\begin{aligned}
\Psi_{\left(v_{1}, v_{2}\right)}\left(x_{1}, \ldots x_{N}\right) & :=\sum_{1 \leq k_{l} \leq 3} \Psi^{k_{1}, \ldots, k_{N}}\left(w_{k_{1}} x_{1}, \ldots w_{k_{N}} x_{N}\right), \\
M_{\left(v_{1}, v_{2}\right)} & :=\left(\alpha_{n}, v_{1} u, v_{2} u^{\prime}, \Psi_{\left(v_{1}, v_{2}\right)}\right)
\end{aligned}
$$

where $w_{1}=v_{1}^{T}, w_{3}=v_{3}=I$ and $w_{2}(x)=v_{2}^{T}\left(x-\alpha_{n} \vec{v}\right)+\alpha_{n} \vec{v}$. We have also forgotten the indices $k$ and $n$ for the sake of simplicity. Obviously, $M_{(I, I)}=M_{k}^{n}$.

Lemma 11. There exists a path $V:[0,1] \rightarrow\left(S \mathcal{O}_{3}(\mathbb{R})\right)^{2}$ such that $V(0)=(I, I)$, $V(1)$ is a critical point of $\left(v_{1}, v_{2}\right) \mapsto \mathcal{E}^{N}\left(M_{\left(v_{1}, v_{2}\right)}\right)$, and $\mathcal{E}^{N}\left(M_{V(t)}\right) \leq \mathcal{E}^{N}\left(M_{I, I}\right)$ for all $t \in[0 ; 1]$.

Proof of Lemma 11 - Since $\Psi_{k}^{n}$ is locally Lipschitz by Theorem 1, one easily shows that $F:\left(v_{1}, v_{2}\right) \mapsto \mathcal{E}^{N}\left(M_{\left(v_{1}, v_{2}\right)}\right)$ is $\mathcal{C}^{2}$ on $\left(S \mathcal{O}_{3}(\mathbb{R})\right)^{2}$. We then simply consider the associated gradient flow to conclude.

Applying this lemma, we may obtain some $X=\left(\alpha_{n}, v u, v^{\prime} u^{\prime}, \tilde{\Psi}\right)$, connected to $M_{k}^{n}$ by a path with a non-increasing energy, and which is a critical point with respect to the rotation of the molecules as defined before. Indeed it is a critical point of the function

$$
\left(H_{1}, H_{2}\right) \mapsto \mathcal{E}^{N}\left(\alpha_{n}, e^{H_{1}} v, e^{H_{2}} v^{\prime},(\tilde{\Psi})_{\left(e^{H_{1}}, e^{H_{2}}\right)}\right)
$$


defined on the space $A_{3}(\mathbb{R}) \times A_{3}(\mathbb{R}), A_{3}(\mathbb{R})$ being the space of $3 \times 3$ antisymmetric real matrices.

Step 2: Expression and expansion of the energy and its derivative. Now, we have

$$
|\tilde{\Psi}|_{\left(e^{\left.H_{1}, e^{H_{2}}\right)}\right.}^{2}=\sum_{1 \leq k_{l} \leq 2}\left|\tilde{\Psi}_{\left(e^{\left.H_{1}, e^{H_{2}}\right)}\right.}^{k_{1}, \ldots, k_{N}}\right|^{2}+\phi
$$

with $\|\phi\|_{H^{1}} \leq C e^{-a \alpha_{n}}$ (by Theorem 2), so we obtain

$$
\begin{aligned}
& \mathcal{E}^{N}\left(\alpha_{n}, e^{H_{1}} v, e^{H_{2}} v^{\prime}, \tilde{\Psi}_{\left(e^{H_{1}}, e^{H_{2}}\right)}\right)= \\
& \sum_{1 \leq k_{l} \leq 2} \mathcal{E}^{N}\left(\alpha_{n}, e^{H_{1}} v, e^{H_{2}} v^{\prime}, \tilde{\Psi}_{\left(e^{H_{1}}, e^{H_{2}}\right)}^{k_{1}, \ldots, k_{N}}\right)+O\left(e^{-a \alpha_{n}}\right) .
\end{aligned}
$$

Let us fix $1 \leq k_{1}, \ldots, k_{N} \leq 2$ and denote $C_{l}=\left\{i, k_{i}=l\right\}, x^{1}=\left(x_{i}\right)_{i \in C_{1}}$, $x^{2}=\left(x_{i}\right)_{i \in C_{2}}, N_{l}=\left|C_{l}\right|$. We then have

$$
\begin{aligned}
& \mathcal{E}^{N}\left(\alpha_{n}, e^{H_{1}} v, e^{H_{2}} v^{\prime}, \tilde{\Psi}_{\left(e^{\left.H_{1}, e^{H_{2}}\right)}\right.}^{k_{1}, \ldots, k_{N}}\right)= \\
&\left\langle H^{N_{1}}(r, z)_{C_{1}} \Psi^{k_{1}, \ldots, k_{N}}, \Psi^{k_{1}, \ldots, k_{N}}\right\rangle+\left\langle H^{N_{2}}\left(r^{\prime}, z^{\prime}\right)_{C_{2}} \Psi^{k_{1}, \ldots, k_{N}}, \Psi^{k_{1}, \ldots, k_{N}}\right\rangle \\
& \quad+\int_{\mathbb{R}^{3 N}}\left|\left(\tau_{-\alpha_{n} \vec{v}}\right)_{x^{2}} \tilde{\Psi}^{k_{1}, \ldots, k_{N}}\right|^{2}\left(\sum_{i \in C_{1}, j \in C_{2}} \frac{1}{\left|\alpha_{n} \vec{v}+e^{H_{2}} x_{j}-e^{H_{1}} x_{i}\right|}\right. \\
& \quad-\sum_{i \in C_{1}} \sum_{j=1}^{m^{\prime}} \frac{z_{j}^{\prime}}{\left|\alpha_{n} \vec{v}+e^{H_{2}} r_{j}-e^{H_{1}} x_{i}\right|}-\sum_{i=1}^{m} \sum_{j \in C_{2}} \frac{z_{i}}{\left|\alpha_{n} \vec{v}+e^{H_{2}} x_{j}-e^{H_{1}} r_{i}\right|} \\
&\left.+\sum_{i=1}^{m} \sum_{j=1}^{m^{\prime}} \frac{z_{i} z_{j}^{\prime}}{\left|\alpha_{n} \vec{v}+e^{H_{2}} r_{j}-e^{H_{1}} r_{i}\right|}\right) d x^{1} d x^{2} .
\end{aligned}
$$

Differentiating this expression with regard to $H=\left(H_{1}, H_{2}\right)$, we find

$$
\begin{aligned}
& d_{H=0} \mathcal{E}^{N}\left(\alpha_{n}, e^{H_{1}} v, e^{H_{2}} v^{\prime}, \tilde{\Psi}_{\left(e^{\left.H_{1}, e^{H_{2}}\right)}\right.}^{k_{1}, \ldots, k_{N}}\right)\left(H_{1}, H_{2}\right)= \\
& -\int_{\mathbb{R}^{3 N}}\left|\left(\tau_{-\alpha_{n} \vec{v}}\right)_{x^{2}} \tilde{\Psi}^{k_{1}, \ldots, k_{N}}\right|^{2}\left(\sum_{i \in C_{1}, j \in C_{2}} \frac{e_{\alpha_{n} \vec{v}+x_{j}-x_{i}} \cdot\left(H_{2} x_{j}-H_{1} x_{i}\right)}{\left|\alpha_{n} \vec{v}+x_{j}-x_{i}\right|^{2}}\right. \\
& -\sum_{i \in C_{1}} \sum_{j=1}^{m^{\prime}} \frac{z_{j}^{\prime} e_{\alpha_{n} \vec{v}+r_{j}-x_{i}} \cdot\left(H_{2} r_{j}-H_{1} x_{i}\right)}{\left|\alpha_{n} \vec{v}+r_{j}-x_{i}\right|^{2}}-\sum_{i=1}^{m} \sum_{j \in C_{2}} \frac{z_{i} e_{\alpha_{n} \vec{v}+x_{j}-r_{i}} \cdot\left(H_{2} x_{j}-H_{1} r_{i}\right)}{\left|\alpha_{n} \vec{v}+x_{j}-r_{i}\right|^{2}} \\
& \left.+\sum_{i=1}^{m} \sum_{j=1}^{m^{\prime}} \frac{z_{i} z_{j}^{\prime} e_{\alpha_{n} \vec{v}+r_{j}-r_{i}} \cdot\left(H_{2} r_{j}-H_{1} r_{i}\right)}{\left|\alpha_{n} \vec{v}+r_{j}-r_{i}\right|^{2}}\right) d x^{1} d x^{2} .
\end{aligned}
$$


Now, we obtain, using lemma 2,

$$
\begin{gathered}
\nabla_{H_{1}=0} \mathcal{E}^{N}\left(\alpha_{n}, e^{H_{1}} v, e^{H_{2}} v^{\prime}, \tilde{\Psi}_{\left(e^{\left.H_{1}, e^{H_{2}}\right)}\right.}^{k_{1}, \ldots, k_{N}}\right)= \\
-\frac{\left(N_{2}-\left|z^{\prime}\right|\right)}{\left(\alpha_{n}\right)^{2}} \int_{\mathbb{R}^{3 N}}\left|\left(\tau_{-\alpha_{n} \vec{v}}\right)_{x^{2}} \tilde{\Psi}^{k_{1}, \ldots, k_{N}}\right|^{2}\left(\sum_{i \in C_{1}} x_{i}-\sum_{i=1}^{m} z_{j} r_{j}\right) \vec{v}^{T} d x^{1} d x^{2} \\
-\frac{\left(N_{2}-\left|z^{\prime}\right|\right)}{\left(\alpha_{n}\right)^{3}} \int_{\mathbb{R}^{3 N}}\left|\left(\tau_{-\alpha_{n} \vec{v}}\right)_{x^{2}} \tilde{\Psi}^{k_{1}, \ldots, k_{N}}\right|^{2}\left(I-3 \vec{v} \vec{v}^{T}\right)\left(\sum_{i \in C_{1}} x_{i} x_{i}^{T}-\sum_{i=1}^{m} z_{i} r_{i} r_{i}^{T}\right) d x^{1} d x^{2} \\
-\frac{1}{\left(\alpha_{n}\right)^{3}} \int_{\mathbb{R}^{3 N}}\left|\left(\tau_{-\alpha_{n} \vec{v}}\right)_{x^{2}} \tilde{\Psi}^{k_{1}, \ldots, k_{N}}\right|^{2}\left(I-3 \vec{v} \vec{v}^{T}\right) . \\
\cdot\left(\sum_{i \in C_{1}} x_{i}-\sum_{i=1}^{m} z_{i} r_{i}\right)\left(\sum_{j \in C_{2}} x_{j}-\sum_{j=1}^{m^{\prime}} z_{j}^{\prime} r_{j}^{\prime}\right)^{T} d x^{1} d x^{2}+O\left(\frac{\int_{\mathbb{R}^{3 N}}\left|\tilde{\Psi}^{k_{1}, \ldots, k_{N}}\right|^{2}}{\left(\alpha_{n}\right)^{4}}\right),
\end{gathered}
$$

and a similar expansion for the gradient with regard to $H_{2}$.

We may now expand the energy with a similar method and obtain

$$
\begin{aligned}
& \mathcal{E}^{N}\left(\alpha_{n}, v, v^{\prime}, \tilde{\Psi}^{k_{1}, \ldots, k_{N}}\right)=\left\langle H^{N_{1}}(r, z)_{C_{1}} \Psi^{k_{1}, \ldots, k_{N}}, \Psi^{k_{1}, \ldots, k_{N}}\right\rangle+ \\
& \left\langle H^{N_{2}}\left(r^{\prime}, z^{\prime}\right)_{C_{2}} \Psi^{k_{1}, \ldots, k_{N}}, \Psi^{k_{1}, \ldots, k_{N}}\right\rangle+\frac{\left(N_{1}-Z_{1}\right)\left(N_{2}-Z_{2}\right)}{\alpha_{n}} \int_{\mathbb{R}^{3 N}}\left|\tilde{\Psi}^{k_{1}, \ldots, k_{N}}\right|^{2} \\
& +\frac{\left(N_{2}-\left|z^{\prime}\right|\right)}{\left(\alpha_{n}\right)^{2}} e_{\vec{v}} \cdot \int_{\mathbb{R}^{3 N}}\left|\left(\tau_{-\alpha_{n} \vec{v}}\right)_{x^{2}} \tilde{\Psi}^{k_{1}, \ldots, k_{N}}\right|^{2}\left(\sum_{i \in C_{1}} x_{i}-\sum_{i=1}^{m} z_{i} r_{i}\right) d x^{1} d x^{2} \\
& -\frac{\left(N_{1}-|z|\right)}{\left(\alpha_{n}\right)^{2}} e_{\vec{v}} \cdot \int_{\mathbb{R}^{3 N}}\left|\left(\tau_{-\alpha_{n} \vec{v}}\right)_{x^{2}} \tilde{\Psi}^{k_{1}, \ldots, k_{N}}\right|^{2}\left(\sum_{j \in C_{2}} x_{j}-\sum_{j=1}^{m^{\prime}} z_{j}^{\prime} r_{j}^{\prime}\right) d x^{1} d x^{2} \\
& -\frac{\left(N_{2}-\left|z^{\prime}\right|\right)}{2\left(\alpha_{n}\right)^{3}} \int_{\mathbb{R}^{3 N}}\left|\left(\tau_{-\alpha_{n} \vec{v}}\right)_{x^{2}} \tilde{\Psi}^{k_{1}, \ldots, k_{N}}\right|^{2}\left(\sum_{i \in C_{1}}\left(I-3 \vec{v} \vec{v}^{T}\right) x_{i} \cdot x_{i}\right. \\
& \left.-\sum_{i=1}^{m} z_{i}\left(I-3 \vec{v} \vec{v}^{T}\right) r_{i} \cdot r_{i}\right) d x^{1} d x^{2} \\
& -\frac{\left(N_{1}-|z|\right)}{2\left(\alpha_{n}\right)^{3}} \int_{\mathbb{R}^{3 N}}\left|\left(\tau_{-\alpha_{n} \vec{v}}\right)_{x^{2}} \tilde{\Psi}^{k_{1}, \ldots, k_{N}}\right|^{2}\left(\sum_{j \in C_{2}}\left(I-3 \vec{v} \vec{v}^{T}\right) x_{j} \cdot x_{j}\right. \\
& \left.-\sum_{j=1}^{m^{\prime}} z_{i}^{\prime}\left(I-3 \vec{v} \vec{v}^{T}\right) r_{j}^{\prime} \cdot r_{j}^{\prime}\right) d x^{1} d x^{2} \\
& +\frac{1}{\left(\alpha_{n}\right)^{3}} \int_{\mathbb{R}^{3 N}}\left|\left(\tau_{-\alpha_{n} \vec{v}}\right)_{x^{2}} \tilde{\Psi}^{k_{1}, \ldots, k_{N}}\right|^{2}\left(I-3 \vec{v} \vec{v}^{T}\right)\left(\sum_{i \in C_{1}} x_{i}-\sum_{i=1}^{m} z_{i} r_{i}\right) . \\
& \left(\sum_{j \in C_{2}} x_{j}-\sum_{j=1}^{m^{\prime}} z_{j}^{\prime} r_{j}^{\prime}\right) d x^{1} d x^{2}+O\left(\frac{\int_{\mathbb{R}^{3 N}}\left|\tilde{\Psi}^{k_{1}, \ldots, k_{N}}\right|^{2}}{\left(\alpha_{n}\right)^{4}}\right) .
\end{aligned}
$$


With a similar computation, it can be proved that

$$
\left|\nabla_{H=0}\left\langle H^{N}\left(R^{n}, Z\right) \tilde{\Psi}^{k_{1}, \ldots, k_{N}}, \tilde{\Psi}^{k_{1}^{\prime}, \ldots, k_{N}^{\prime}}\right\rangle\right|=O\left(e^{-a \alpha_{n}}\right)
$$

when $k_{l}^{\prime}=3$ for some $l$.

As a conclusion, we obtain, by summing these expressions,

$$
\mathcal{E}^{N}\left(\alpha_{n}, e^{H_{1}} v, e^{H_{2}} v^{\prime}, \tilde{\Psi}_{k}^{n}\right)=A_{n}-\frac{B_{n}}{\alpha_{n}}+\frac{C_{n}}{\left(\alpha_{n}\right)^{2}}+\frac{D_{n}+E_{n}}{\left(\alpha_{n}\right)^{3}}+O\left(\frac{1}{\left(\alpha_{n}\right)^{4}}\right)
$$

where

$$
\begin{gathered}
A_{n}=\sum_{N_{1}+N_{2}=N} \sum_{\left|C_{1}\right|=N_{1}}\left\langle\left(H^{N_{1}}(r, z)_{C_{1}}+H^{N_{2}}\left(r^{\prime}, z^{\prime}\right)_{C_{2}}\right) \Psi^{C_{1}, C_{2}}, \Psi^{C_{1}, C_{2}}\right\rangle \\
B_{n}=-\sum_{N_{1}+N_{2}=N} \sum_{\left|C_{1}\right|=N_{1}}\left(N_{1}-|z|\right)\left(N_{2}-\left|z^{\prime}\right|\right)\left\|\tilde{\Psi}^{C_{1}, C_{2}}\right\|_{L^{2}}^{2} \geq 0
\end{gathered}
$$

and with similar definitions for $C_{n}, D_{n}, E_{n}$ ( $D_{n}$ contains the two terms involving $\left(N_{1}-|z|\right)$ and $\left(N_{2}-\left|z^{\prime}\right|\right)$, and $E_{n}$ is the third term).

Step 3: A boot-strap argument. We have

$$
\begin{aligned}
A_{n} \geq & \sum_{N_{1}+N_{2}=N}\left(E^{N_{1}}(r, z)+E^{N_{2}}\left(r^{\prime}, z^{\prime}\right)\right) \sum_{\left|C_{1}\right|=N_{1}}\left\|\tilde{\Psi}^{C_{1}, C_{2}}\right\|_{L^{2}}^{2} \\
= & c \sum_{\left|C_{1}\right|=|z|}\left\|\tilde{\Psi}^{C_{1}, C_{2}}\right\|_{L^{2}}^{2} \\
& +\sum_{\substack{N_{1}+N_{2}=N \\
N_{1} \neq|z|}}\left(E^{N_{1}}(r, z)+E^{N_{2}}\left(r^{\prime}, z^{\prime}\right)\right) \sum_{\left|C_{1}\right|=N_{1}}\left\|\tilde{\Psi}^{C_{1}, C_{2}}\right\|_{L^{2}}^{2} \\
= & c+O\left(e^{-a \alpha_{n}}\right)+\sum_{\substack{N_{1}+N_{2}=N \\
N_{1} \neq|z|}}\left(E^{N_{1}}(r, z)+E^{N_{2}}\left(r^{\prime}, z^{\prime}\right)-c\right) \sum_{\left|C_{1}\right|=N_{1}}\left\|\tilde{\Psi}^{C_{1}, C_{2}}\right\|_{L^{2}}^{2} \\
\geq & c+O\left(e^{-a \alpha_{n}}\right)+\epsilon \sum_{\substack{N_{1}+N_{2}=N \\
N_{1} \neq|z|}} \| \tilde{C}_{1} \mid=N_{1} \\
\geq & c+\epsilon^{\prime} B_{n}+O\left(e^{-a \alpha_{n}}\right)
\end{aligned}
$$

where $\epsilon=\min \left\{E^{N_{1}}(r, z)+E^{N_{2}}\left(r^{\prime}, z^{\prime}\right), N_{1}+N_{2}=N, N_{1} \neq|z|\right\}-c>0$ (H1). Now, we have by the definition of $M_{k}^{n}$ and $X_{k}^{n}$,

$$
\mathcal{E}^{N}\left(\alpha_{n}, e^{H_{1}} v, e^{H_{2}} v^{\prime}, \tilde{\Psi}_{k}^{n}\right)<c
$$

so we obtain, for $n$ large enough,

$$
0 \leq \frac{\epsilon^{\prime}}{2} B_{n} \leq\left(\epsilon^{\prime}-\frac{1}{\alpha_{n}}\right) B_{n} \leq-\frac{C_{n}}{\left(\alpha_{n}\right)^{2}}-\frac{D_{n}+E_{n}}{\left(\alpha_{n}\right)^{3}}+O\left(\frac{1}{\left(\alpha_{n}\right)^{4}}\right) .
$$

Using the fact that $\tilde{\Psi}_{k}^{n}$ converges to some critical point at infinity, one easily sees that $A_{n}, B_{n}, C_{n}$ and $D_{n}$ are bounded as $n$ goes to $+\infty$, and finally $B_{n}=$ 
$O\left(\frac{1}{\left(\alpha_{n}\right)^{2}}\right)$. But we may write for instance, for $1 \leq k_{l} \leq 2$ with $\left|C_{1}\right|:=N_{1} \neq|z|$, by $(14)$

$$
\begin{gathered}
\left.\left|\left(N_{2}-\left|z^{\prime}\right|\right) e_{\vec{v}} \cdot \int_{\mathbb{R}^{3 N}}\right|\left(\tau_{-\alpha_{n} \vec{v}}\right)_{x^{2}} \tilde{\Psi}^{k_{1}, \ldots, k_{N}}\right|^{2}\left(\sum_{i \in C_{1}} x_{i}-\sum_{i=1}^{m} z_{i} r_{i}\right) d x^{1} d x^{2} \mid \\
\leq\left|N_{2}-\right| z^{\prime}||\left(N_{1}+|z|\right) \alpha_{n} \int_{\mathbb{R}^{3 N}}\left|\left(\tau_{-\alpha_{n} \vec{v}}\right)_{x^{2}} \tilde{\Psi}^{k_{1}, \ldots, k_{N}}\right|^{2} d x^{1} d x^{2} \\
=\alpha_{n}\left|N_{2}-\right| z^{\prime}|| O\left(\left\|\tilde{\Psi}^{k_{1}, \ldots, k_{N}}\right\|_{L^{2}}^{2}\right)
\end{gathered}
$$

and so one can easily prove

$$
C_{n}=O\left(\alpha_{n} B_{n}\right), \quad D_{n}=O\left(\left(\alpha_{n}\right)^{2} B_{n}\right) .
$$

Now $C_{n}=O\left(\frac{1}{\alpha_{n}}\right)$ and by (14), $B_{n}=O\left(\frac{1}{\left(\alpha_{n}\right)^{3}}\right)$. Using one more time (15), we obtain $C_{n}=O\left(\frac{1}{\left(\alpha_{n}\right)^{2}}\right)$ and $D_{n}=O\left(\frac{1}{\alpha_{n}}\right)$. As a consequence,

$$
\mathcal{E}^{N}\left(\alpha_{n}, e^{H_{1}} v, e^{H_{2}} v^{\prime}, \tilde{\Psi}_{k}^{n}\right)=A_{n}+\frac{E_{n}}{\left(\alpha_{n}\right)^{3}}+O\left(\frac{1}{\left(\alpha_{n}\right)^{4}}\right) .
$$

Using the same estimates, we also obtain an expansion of the form

$$
\nabla_{H_{1}=0} \mathcal{E}^{N}\left(\alpha_{n}, e^{H_{1}} v, e^{H_{2}} v^{\prime}, \tilde{\Psi}_{\left(H_{1}, H_{2}\right)}\right)=\frac{E_{n}^{\prime}}{\left(\alpha_{n}\right)^{3}}+O\left(\frac{1}{\left(\alpha_{n}\right)^{4}}\right)=0
$$

where $E_{n}^{\prime}$ corresponds to the derivative of the dipole/dipole term. But now it is known that

$$
\mathcal{P}_{\mathcal{A}_{3}(\mathbb{R})^{\perp}}\left[\nabla_{H_{1}=0} \mathcal{E}^{N}\left(\alpha_{n}, e^{H_{1}} v, e^{H_{2}} v^{\prime}, \tilde{\Psi}_{\left(H_{1}, H_{2}\right)}\right)\right]=0
$$

where $\mathcal{P}_{\mathcal{A}_{3}(\mathbb{R})^{\perp}}$ is the orthogonal projection onto $\mathcal{A}_{3}(\mathbb{R})^{\perp}$. We thus have $\mathcal{P}_{\mathcal{A}_{3}(\mathbb{R})^{\perp}} E_{n}^{\prime}=$ $O\left(\frac{1}{\alpha_{n}}\right)$ and so $\mathcal{P}_{\mathcal{A}_{3}(\mathbb{R}) \perp} E_{n}^{\prime} \rightarrow 0$ as $n$ goes to $+\infty$. A same result holds for the derivative with respect to $H_{2}$. Recall now that in fact $v=v_{k}^{n}, v^{\prime}=v_{k}^{\prime n}, u=u_{k}^{n}$ and $u^{\prime}=u_{k}^{\prime n}$. As above we may assume by extracting a sequence if necessary that $v_{k}^{n}$ and $v_{k}^{\prime n}$ converge to some $v_{k}$ and $v_{k}^{\prime}$ as $n \rightarrow+\infty$. $\tilde{\Psi}_{k}^{n}$,

We may now pass to the limit and obtain, by (14) and the convergence of

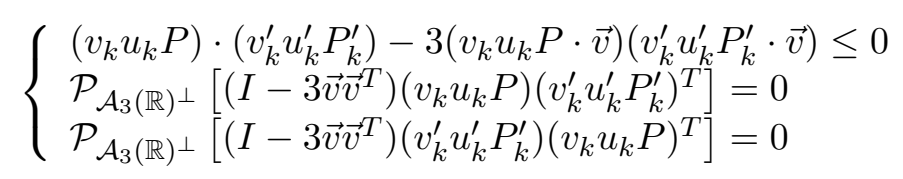

for $k \in\{1,2\}$, and where $P:=P_{\psi}$ and $P_{k}:=P_{\psi_{k}^{\prime}}$.

Step 4: Study of the critical points of the dipole/dipole interaction. We have the following result, which is proved in Appendix 2. 
Lemma 12. Let be $P, P^{\prime} \in \mathbb{R}^{3} \backslash\{0\}$ and $e \in S^{2}$. The critical points of the function

$F_{P, P^{\prime}}^{e}:\left(u, u^{\prime}\right) \mapsto(u P) \cdot\left(u^{\prime} P^{\prime}\right)-3(u P \cdot e)\left(u^{\prime} P^{\prime} \cdot e\right)=\left(\left(I-3 e e^{T}\right)(u P)\right) \cdot\left(u^{\prime} P^{\prime}\right)$

defined on $\mathrm{SO}_{3}(\mathbb{R})^{2}$ are given by

1. $u_{0} P=\epsilon|P| e, u_{0}^{\prime} P^{\prime}=\epsilon\left|P^{\prime}\right| e$ with $\epsilon= \pm 1$. Then $\left(u_{0}, u_{0}^{\prime}\right)$ is a minimum of $F$, and $F_{P, P^{\prime}}^{e}\left(u_{0}, u_{0}^{\prime}\right)=-2|P|\left|P^{\prime}\right|$.

2. $u_{4} P=\epsilon|P| e, u_{4}^{\prime} P^{\prime}=-\epsilon\left|P^{\prime}\right| e$ with $\epsilon= \pm 1$. Then $\left(u_{4}, u_{4}^{\prime}\right)$ is a maximum of $F$ with a Morse index equal to 4 , and $F_{P, P^{\prime}}^{e}\left(u_{4}, u_{4}^{\prime}\right)=2|P|\left|P^{\prime}\right|$.

3. $u_{1} P=|P| v, u_{1}^{\prime} P^{\prime}=-\left|P^{\prime}\right| v$ for some $v \in S^{2} \cap(e)^{\perp}$. Then $\left(u_{1}, u_{1}^{\prime}\right)$ has a Morse index equal to 1 , and $F_{P, P^{\prime}}^{e}\left(u_{1}, u_{1}^{\prime}\right)=-|P|\left|P^{\prime}\right|$.

4. $u_{2} P=|P| v, u_{2}^{\prime} P^{\prime}=\left|P^{\prime}\right| v$ for some $v \in S^{2} \cap(e)^{\perp}$. Then $\left(u_{2}, u_{2}^{\prime}\right)$ has a Morse index equal to 2 , and $F_{P, P^{\prime}}^{e}\left(u_{1}, u_{1}^{\prime}\right)=|P|\left|P^{\prime}\right|$.

\begin{tabular}{|c|c|c|c|}
\hline Index & Energy & & \\
\hline 4 & $2|P|\left|P^{\prime}\right|$ & $\rightarrow \longleftarrow$ & $\longleftarrow \rightarrow$ \\
\hline 2 & $|P|\left|P^{\prime}\right|$ & & \\
\hline 1 & $-|P|\left|P^{\prime}\right|$ & & $\rightarrow$ \\
\hline 0 & $-2|P|\left|P^{\prime}\right|$ & $\rightarrow \quad \rightarrow$ & $\longleftarrow \leftarrow$ \\
\hline
\end{tabular}

Figure 4: The critical points of the dipole/dipole interaction

By (16), $\left(v_{k} u_{k}, v_{k}^{\prime} u_{k}^{\prime}\right)$ is a critical point of $F_{P, P_{k}^{\prime}}^{\vec{v}}$, with a non-positive energy. Since $P \neq 0$ and $P_{k}^{\prime} \neq 0(H 1)$, we conclude by Lemma 12 that $F_{P, P_{k}^{\prime}}^{\vec{v}}\left(v_{k} u_{k}, v_{k}^{\prime} u_{k}^{\prime}\right) \leq$ $-|P|\left|P_{k}^{\prime}\right|<0$.

As a consequence, applying Lemma 2, we obtain

$$
\begin{aligned}
\mathcal{E}^{N}\left(\alpha_{n}, v_{k}^{n} u_{k}^{n}, v_{k}^{\prime n} u_{k}^{\prime n},\left(v_{k}^{n} u_{k}^{n} \cdot \psi\right) \wedge\right. & \left.\left(\tau_{\alpha_{n} \vec{v}} \cdot v_{k}^{\prime n} u_{k}^{\prime n} \cdot \psi_{k}^{\prime}\right)\right)= \\
& c+\frac{F_{P, P_{k}^{\prime}}^{(}\left(v_{k}^{n} u_{k}^{n}, v_{k}^{\prime n} u_{k}^{\prime n}\right)}{\left(\alpha_{n}\right)^{3}}+O\left(\frac{1}{\left(\alpha_{n}\right)^{4}}\right)<c
\end{aligned}
$$

for $n$ large enough, since

$$
\lim _{n \rightarrow+\infty} F_{P, P_{k}^{\prime}}^{\vec{v}}\left(v_{k}^{n} u_{k}^{n}, v_{k}^{\prime n} u_{k}^{\prime n}\right)=F_{P, P_{k}^{\prime}}^{\vec{v}}\left(v_{k} u_{k}, v_{k}^{\prime}{ }_{k}^{\prime}{ }_{k}\right)<0 .
$$


Let us now denote by

$$
\tilde{M}_{k}^{n}:=\left(\alpha_{n}, v_{k}^{n} u_{k}^{n}, v_{k}^{\prime n} u_{k}^{\prime n},\left(v_{k}^{n} u_{k}^{n} \cdot \psi\right) \wedge\left(\tau_{\alpha_{n} \vec{v}} \cdot v_{k}^{\prime n} u_{k}^{\prime n} \cdot \psi_{k}^{\prime}\right)\right) .
$$

For $n$ large enough, we may now construct a path connecting $X_{k}^{n}$ to $\tilde{M}_{k}^{n}$ $k \in\{1,2\}$, with an energy below $c$, by Lemma 10 .

Step 5: Connecting the two tensor products. To end the proof, it remains to connect $M_{1}^{n}$ and $M_{2}^{n}$ by a path with a maximum energy below $c$. We fix here $n$ and forget this subscript. Let us now introduce the function $F$ defined by

$$
F\left(\nu, \nu^{\prime}, \phi\right)=F_{P, P_{\phi}}^{\vec{v}}\left(\nu, \nu^{\prime}\right)
$$

for $\left(\nu, \nu^{\prime}, \phi\right) \in W:=S \mathcal{O}_{3}(\mathbb{R})^{2} \times V$ where $V$ is the unit sphere of the finite dimensional space $\operatorname{ker}\left(H^{\left|z^{\prime}\right|}\left(r^{\prime}, z^{\prime}\right)-E^{\left|z^{\prime}\right|}\left(r^{\prime}, z^{\prime}\right)\right)$. We now denote by $Y_{k}:=$ $\left(u_{k} v_{k}, u_{k}^{\prime} v_{k}^{\prime}, \psi_{k}\right)$ for $k \in\{1,2\}$. Notice that we have shown

$$
\max \left(F\left(Y_{1}\right), F\left(Y_{2}\right)\right) \leq-\min _{\phi \in V}\left\{|P|\left|P_{\phi}\right|\right\}<0 .
$$

Now, let be

$$
\tilde{c}:=\inf _{Y \in \Gamma^{\prime}} \max _{t \in[0,1]} F(Y(t))
$$

where

$$
\Gamma^{\prime}=\left\{Y \in \mathcal{C}^{0}([0,1], W), Y(0)=Y_{1}, Y(1)=Y_{2}\right\} .
$$

We have the following

Lemma 13. We have $\tilde{c} \leq-\min _{\phi \in V}\left\{|P|\left|P_{\phi}\right|\right\}:=C<0$.

Proof of Lemma 13 - Suppose that $\tilde{c}>\max \left(F\left(Y_{1}\right), F\left(Y_{2}\right)\right)$. By the methods of [14], we may find a sequence $y_{n} \in W$ such that $F\left(y_{n}\right) \rightarrow \tilde{c}, \nabla F\left(y_{n}\right) \rightarrow 0$ and $d_{\left(\nu, \nu^{\prime}\right)}^{2} F \geq-e_{n}$ on a space of codimension at most 1 , with $e_{n} \rightarrow 0$ as $n \rightarrow+\infty$. $W$ being compact, up to a subsequence, $y_{n}=\left(\nu_{n}, \nu_{n}^{\prime}, \phi_{n}\right)$ converges to a critical point $y=\left(\nu, \nu^{\prime}, \phi\right)$ of energy $\tilde{c}$, and with a Morse index with respect to $\left(\nu, \nu^{\prime}\right)$ variations at most 1 . By Lemma 12, we necessary have $F(y) \leq-|P|\left|P_{\phi}\right|$.

Let $Y_{n} \in \Gamma^{\prime}$ be a path connecting $Y_{1}$ and $Y_{2}$ such that $\max _{t \in[0 ; 1]} F(Y(t)) \leq$ $\tilde{c}+\frac{1}{\alpha_{n}}$. We then have

$$
\begin{aligned}
& \mathcal{E}^{N}\left(\alpha_{n}, \nu_{n}(t), \nu_{n}^{\prime}(t),\left(\nu_{n}(t) \cdot \psi\right)\right.\left.\wedge\left(\tau_{\alpha_{n} \vec{v}} \cdot \nu_{n}^{\prime}(t) \cdot \psi_{n}(t)\right)\right) \\
&=c+\frac{F\left(Y_{n}(t)\right)}{\left(\alpha_{n}\right)^{3}}+O\left(\frac{1}{\left(\alpha_{n}\right)^{4}}\right) \leq c+\frac{C}{\left(\alpha_{n}\right)^{3}}+O\left(\frac{1}{\left(\alpha_{n}\right)^{4}}\right)<c
\end{aligned}
$$

for $n$ large enough.

As a conclusion, we have built a path $\tilde{\gamma}_{n}$ for $n$ large enough such that

$$
\max _{t \in[0 ; 1]} \mathcal{E}^{N}\left(\tilde{\gamma}_{n}(t)\right)<c
$$

which is a contradiction and ends the proof of Theorem 7 . 


\section{Appendix 1: Non-isotropic exponential decay of the electronic density}

We want to prove here Lemma 3 , that we recall for the reader's convenience.

Lemma 14. Let $\Psi_{R}$ be an eigenfunction associated to the eigenvalue $\lambda_{d}^{N}(R, Z)$ and $\rho_{R}$ be the electronic density. We introduce $\epsilon_{R}=\Sigma^{N}(R, Z)-\lambda_{d}^{N}(R, Z)$. Then

1. $\rho_{R}$ satisfies the inequation

$$
-\frac{1}{2} \Delta \rho_{R}+V_{R} \rho_{R}+\epsilon_{R} \rho_{R} \leq 0
$$

2. With $R_{1}(\epsilon):=\max \left(R_{0}+1, R_{0}+\frac{2 N p}{\epsilon}\right)$ and $C(\epsilon):=\cup_{j=1}^{p}\left\{x,\left|x-X_{j}\right|=\right.$ $\left.R_{1}(\epsilon)\right\}$, and if $r>2 R_{1}\left(\epsilon_{R}\right)$, then we have

$$
\begin{aligned}
\rho_{R}(x) & \leq\left\|\rho_{R}\right\|_{L^{\infty}\left(C\left(\epsilon_{R}\right)\right)} \sum_{j=1}^{p} e^{-\sqrt{\epsilon_{R} / p}\left(\left|X_{j}-x\right|-R_{1}\left(\epsilon_{R}\right)\right)} \\
& \leq p\left\|\rho_{R}\right\|_{L^{\infty}\left(C\left(\epsilon_{R}\right)\right)} e^{-\sqrt{\epsilon_{R} / p}\left(\delta(x)-R_{1}\left(\epsilon_{R}\right)\right)}, \\
& \leq M e^{-\sqrt{\epsilon_{R} / p}\left(\delta(x)-R_{1}\left(\epsilon_{R}\right)\right)}
\end{aligned}
$$

on $\mathcal{U}\left(R_{1}\left(\epsilon_{R}\right)\right)$, where $\delta(x)=\min \left\{\left|x-X_{j}\right|, j=1, \ldots, d\right\}$, and $M=$ $M\left(p, N, R_{1}\left(\epsilon_{R}\right)\right)$.

Proof - The fact that $\rho_{R}$ is a solution to (10) is essentially proved in [16] (the proof is written for atoms with no nuclei/nuclei interaction).

The proof of (19) follows ideas of [15]. We have

$$
V_{R}(x) \geq-\sum_{j=1}^{p} \frac{N}{\left|x-X_{j}\right|-R_{0}}
$$

for all $x \in \mathcal{U}\left(R_{1}\left(\epsilon_{R}\right)\right)$, so that $\rho_{R}$ satisfies

$$
-\frac{1}{2} \Delta \rho_{R}+\sum_{j=1}^{p}\left(\frac{\epsilon_{R}}{p}-\frac{N}{\left|x-X_{j}\right|-R_{0}}\right) \rho_{R} \leq 0 .
$$

on $\mathcal{U}$. Let $f$ be the radial positive solution of

$$
-\frac{1}{2} \Delta f+\left(\frac{\epsilon_{R}}{p}-\frac{N}{|x|-R_{0}}\right) f=0
$$

on $\mathbb{R}^{3} \backslash B\left(0, R_{1}\left(\epsilon_{R}\right)\right)$, such that $f(x)=1$ if $|x|=R_{1}\left(\epsilon_{R}\right)$ ( $f$ is a Whittaker function, see [15]). Then $f_{j}(x)=f\left(x-X_{j}\right)$ is a positive solution of

$$
-\frac{1}{2} \Delta f_{j}+\left(\frac{\epsilon_{R}}{p}-\frac{N}{\left|x-X_{j}\right|-R_{0}}\right) f_{j}=0
$$


on $\mathbb{R}^{3} \backslash B\left(X_{j}, R_{1}\left(\epsilon_{R}\right)\right)$ and $F=\sum_{j=1}^{p} f_{j}$ is a solution of

$$
-\frac{1}{2} \Delta F+\sum_{j=1}^{p}\left(\frac{\epsilon_{R}}{p}-\frac{N}{\left|x-X_{j}\right|-R_{0}}\right) F=0 .
$$

such that $F(x) \geq 1$ if $x \in C\left(\epsilon_{R}\right)$.

Since $\rho_{R} \in \mathcal{C}^{\infty}\left(\mathcal{U}\left(R_{1}\left(\epsilon_{R}\right)\right)\right)$ and $\lim _{|x| \rightarrow+\infty} \rho_{R}(x)=0$, the maximum principle implies

$$
\rho_{R} \leq\left\|\rho_{R}\right\|_{L^{\infty}\left(C\left(\epsilon_{R}\right)\right)} F(x) .
$$

But, on $\mathbb{R}^{3} \backslash B\left(X_{j}, R_{1}\left(\epsilon_{R}\right)\right)$, we also have

$$
\frac{\epsilon_{R}}{p}-\frac{N}{\left|x-X_{j}\right|-R_{0}} \geq \frac{\epsilon_{R}}{2 p}>0,
$$

so that (maximum principle)

$$
f_{j}(x) \leq \frac{R_{1}\left(\epsilon_{R}\right)}{\left|X_{i}-x\right|} e^{-\sqrt{\epsilon_{R} / p}\left(\left|x-X_{j}\right|-R_{1}\left(\epsilon_{R}\right)\right)} \leq e^{-\sqrt{\epsilon_{R} / p}\left(\left|x-X_{j}\right|-R_{1}\left(\epsilon_{R}\right)\right)}
$$

on $\mathbb{R}^{3} \backslash B\left(X_{j}, R_{1}\left(\epsilon_{R}\right)\right)$ which implies

$$
\begin{aligned}
F(x) & \leq \sum_{j=1}^{p} e^{-\sqrt{\epsilon_{R} / p}\left(\left|x-X_{j}\right|-R_{1}\left(\epsilon_{R}\right)\right)} \\
& \leq p e^{-\sqrt{\epsilon_{R} / p}\left(\frac{1}{p} \sum_{j=1}^{p}\left|x-X_{j}\right|-R_{1}\left(\epsilon_{R}\right)\right)} \\
& \leq p e^{-\sqrt{\epsilon_{R} / p}\left(\delta(x)-R_{1}\left(\epsilon_{R}\right)\right)} .
\end{aligned}
$$

To end the proof, we now remark that since $\rho_{R}$ is real analytic on $\mathcal{U}\left(R_{0}+1 / 2\right)$, there exists a constant $M$ depending only on $R_{1}\left(\epsilon_{R}\right)$ such that

$$
\left\|\rho_{R}\right\|_{L^{\infty}\left(C\left(\epsilon_{R}\right)\right)} \leq M\left\|\rho_{R}\right\|_{\left.L^{1}\left(\mathcal{U}\left(R_{0}+1 / 2\right)\right)\right)} \leq M\left\|\rho_{R}\right\|_{L^{1}\left(\mathbb{R}^{3}\right)}=M \cdot N
$$

and so

$$
\rho_{R} \leq M N p e^{-\sqrt{\epsilon_{R} / p}\left(\delta(x)-R_{1}\left(\epsilon_{R}\right)\right)} .
$$




\section{Appendix 2: Study of the critical points of the dipole/dipole interac- tion}

We prove here Lemma 12 that we recall.

Lemma 15. Let be $P, P^{\prime} \in \mathbb{R}^{3} \backslash\{0\}$ and $e \in S^{2}$. The critical points of the function

$$
F_{P, P^{\prime}}^{e}:\left(u, u^{\prime}\right) \mapsto(u P) \cdot\left(u^{\prime} P^{\prime}\right)-3(u P \cdot e)\left(u^{\prime} P^{\prime} \cdot e\right)=\left(\left(I-3 e e^{T}\right)(u P)\right) \cdot\left(u^{\prime} P^{\prime}\right)
$$

defined on $\mathrm{SO}_{3}(\mathbb{R})^{2}$ are given by

1. $u_{0} P=\epsilon|P| e, u_{0}^{\prime} P^{\prime}=\epsilon\left|P^{\prime}\right| e$ with $\epsilon= \pm 1$. Then $\left(u_{0}, u_{0}^{\prime}\right)$ is a minimum of $F$, and $F_{P, P^{\prime}}^{e}\left(u_{0}, u_{0}^{\prime}\right)=-2|P|\left|P^{\prime}\right|$.

2. $u_{4} P=\epsilon|P| e, u_{4}^{\prime} P^{\prime}=-\epsilon\left|P^{\prime}\right| e$ with $\epsilon= \pm 1$. Then $\left(u_{4}, u_{4}^{\prime}\right)$ is a maximum of $F$ with a Morse index equal to 4 , and $F_{P, P^{\prime}}^{e}\left(u_{4}, u_{4}^{\prime}\right)=2|P|\left|P^{\prime}\right|$.

3. $u_{1} P=|P| v, u_{1}^{\prime} P^{\prime}=-\left|P^{\prime}\right| v$ for some $v \in S^{2} \cap(e)^{\perp}$. Then $\left(u_{1}, u_{1}^{\prime}\right)$ has a Morse index equal to 1 , and $F_{P, P^{\prime}}^{e}\left(u_{1}, u_{1}^{\prime}\right)=-|P|\left|P^{\prime}\right|$.

4. $u_{2} P=|P| v, u_{2}^{\prime} P^{\prime}=\left|P^{\prime}\right| v$ for some $v \in S^{2} \cap(e)^{\perp}$. Then $\left(u_{2}, u_{2}^{\prime}\right)$ has a Morse index equal to 2 , and $F_{P, P^{\prime}}^{e}\left(u_{1}, u_{1}^{\prime}\right)=|P|\left|P^{\prime}\right|$.

Proof - It is simpler to study the function

$$
(x, y) \mapsto\left|P \| P^{\prime}\right| x \cdot y-3(x \cdot e)(y \cdot e)
$$

defined on $S^{2} \times S^{2}$, but we shall not use here this expression, to keep the point of view imposed by the proof of Theorem 7 .

If $\left(u, u^{\prime}\right)$ is a critical point of $F_{P, P^{\prime}}^{e}$, then $(0,0)$ is a critical point of $f$ : $\left(H, H^{\prime}\right) \mapsto F_{u P, u^{\prime} P^{\prime}}^{e}\left(e^{H}, e^{H^{\prime}}\right)$, defined on $\mathcal{A}_{3}(\mathbb{R}) \times \mathcal{A}_{3}(\mathbb{R})$. We may assume $u=$ $u^{\prime}=I$ and $|P|=\left|P^{\prime}\right|=1$ to simplify notations, and we denote by $M:=I-3 e e^{T}$. Then we have

$$
\left\{\begin{array} { l } 
{ M P ^ { \prime } P ^ { T } \in \mathcal { A } _ { 3 } ( \mathbb { R } ) ^ { \perp } } \\
{ M P ( P ^ { \prime } ) ^ { T } \in \mathcal { A } _ { 3 } ( \mathbb { R } ) ^ { \perp } }
\end{array} \Longleftrightarrow \left\{\begin{array}{l}
M P^{\prime} P^{T}=P\left(P^{\prime}\right)^{T} M \\
M P\left(P^{\prime}\right)^{T}=P^{\prime} P^{T} M
\end{array}\right.\right.
$$

Multiplying by $M$, we find for instance $M^{2} P^{\prime} P^{T}=P^{\prime} P^{T} M^{2}$ and so $M^{2} P^{\prime}=$ $\left(P^{T} M^{2} P\right) P^{\prime}$, showing that $P$ and $P^{\prime}$ are eigenvectors of $M^{2}$. It is then easy to see that they are eigenvectors of $M$. Using (20), we obtain $P= \pm P^{\prime}$. The critical points are thus those given in the Lemma.

The second derivative is given by

$$
d^{2} f_{P, P^{\prime}}\left(H, H^{\prime}\right)=2(M H P) \cdot\left(H^{\prime} P^{\prime}\right)+\left(M H^{2} P\right) \cdot P^{\prime}+\left(M\left(H^{\prime}\right)^{2} P^{\prime}\right) \cdot P .
$$

Suppose for instance that $P=e$. Let $\left(e_{2}, e_{3}\right)$ be an orthogonal basis of $e^{\perp}$. Then, in the basis $\left(e_{1}=e, e_{2}, e_{3}\right), M=\operatorname{diag}(-2,1,1)$. We introduce $H_{i j}:=$ 
$e_{i} e_{j}^{T}-e_{j} e_{i}^{T}$, so that $\left(H_{i j}\right)_{1 \leq i<j \leq 3}$ is an orthogonal basis of $\mathcal{A}_{3}(\mathbb{R})$. The matrix of $d_{P, P}^{2} f$ is this basis is then

$$
d_{P, P}^{2} f=\left(\begin{array}{cccccc}
2 & 0 & 0 & 1 & 0 & 0 \\
0 & 0 & 0 & 0 & 0 & 0 \\
0 & 0 & 2 & 0 & 0 & 1 \\
1 & 0 & 0 & 2 & 0 & 0 \\
0 & 0 & 0 & 0 & 0 & 0 \\
0 & 0 & 1 & 0 & 0 & 2
\end{array}\right) \sim \operatorname{diag}(1,1,3,3,0,0)
$$

and obviously $d_{P,-P}^{2} f \sim \operatorname{diag}(-1,-1,-3,-3,0,0)$.

If $P \perp e$, we use the same basis $\left(H_{i j}\right)_{1 \leq i<j \leq 3}$ but with $e_{2}=P$ and find

$$
d_{P, P}^{2} f=\left(\begin{array}{cccccc}
-1 & 0 & 0 & -2 & 0 & 0 \\
0 & -1 & 0 & 0 & 1 & 0 \\
0 & 0 & 0 & 0 & 0 & 0 \\
-2 & 0 & 0 & -1 & 0 & 0 \\
0 & -2 & 0 & 0 & -1 & 0 \\
0 & 0 & 0 & 0 & 0 & 0
\end{array}\right) \sim \operatorname{diag}(1,-2,-3,0,0,0),
$$

and obviously $d_{P,-P}^{2} f \sim \operatorname{diag}(-1,2,3,0,0,0)$. 


\section{References}

[1] S. Agmon. Lectures on exponential decay of solutions of second-order elliptic equations. Princeton University Press, 1982.

[2] A. Bahri. Critical Points at Infinity in some Variational Problems. Pitman Research Notes in Mathematics Series, 182. Longman Scientific and Technical Ed., 1989.

[3] E. Cancès et al. Computational quantum chemistry: a primer. Handbook of numerical analysis, Vol. X, 3-270, 2003.

[4] I. Catto, P.-L. Lions. Binding of atoms and stability of molecules in Hartree and Thomas-Fermi type theories. Part I. Comm. Part. Diff. Eq., 17 (7-8) : 1051-1110, 1992.

[5] I. Catto, P.-L. Lions. Binding of atoms and stability of molecules in Hartree and Thomas-Fermi type theories. Part II. Comm. Part. Diff. Eq., 18 (1-2) : 305-354, 1993.

[6] I. Catto, P.-L. Lions. Binding of atoms and stability of molecules in Hartree and Thomas-Fermi type theories. Part III. Comm. Part. Diff. Eq., 18 (3-4) : 381-429, 1993.

[7] I. Catto, P.-L. Lions. Binding of atoms and stability of molecules in Hartree and Thomas-Fermi type theories. Part IV. Comm. Part. Diff. Eq., 18 (7-8) : 1149-1159, 1993.

[8] G. Fang, N. Ghoussoub. Morse-type information on Palais-Smale sequences obtained by min-max principles. Manuscripta Math., 75 : 81-95, 1992.

[9] J. B. Foresman, A. Frisch. Exploring Chemistry with Electronic Structure Methods, Gaussian Inc, 1996. Chapter 3 and references therein.

[10] G. Friesecke. The multiconfiguration equations for atoms and molecules: charge quantization and existence of solutions. Mathematical Institute, University of Oxford, U.K., 1999, preprint (to appear in Arch. Rat. Mech. Analysis).

[11] S. Fournais, M. and T. Hoffmann-Ostenhof, T. Østergaard Sørensen. The Electron Density is Smooth Away from the Nuclei. Commun. Math. Phys., 228 : 401-415, 2002.

[12] S. Fournais, M. and T. Hoffmann-Ostenhof, T. Østergaard Sørensen. On the regularity of the density of electronic wavefunctions. Mathematical results in quantum mechanics (Taxco, 2001), 143-148, Contemp. Math., 307, 2002.

[13] S. Fournais, M. and T. Hoffmann-Ostenhof, T. Østergaard Sørensen. Analyticity of the Density of Electronic Wavefunctions. Preprint, 2002. 
[14] N. Ghoussoub. Duality and perturbation methods in critical point theory. Cambridge University Press, 1993.

[15] M. and T. Hoffmann-Ostenhof. "Schrödinger inequalities" and asymptotic behavior of the electron density of atoms and molecules. Phys. Rev. A, 16 (5) : 1782-1785, 1977.

[16] M. and T. Hoffmann-Ostenhof, T. Østergaard Sørensen. Electron Wavefunctions and densities for atoms. Ann. Henri Poincaré, 2 : 77-100, 2001.

[17] M. and T. Hoffmann-Ostenhof, H. Stremnitzer. Local properties of Coulombic wave functions. Commun. Math. Phys., 163 : 185-215, 1994.

[18] W. Hunziker. On the Spectra of Schrödinger Multiparticle Hamiltonians. Helv. Phys. Acta, 39 : 451-462, 1966.

[19] W. Hunziker, I.M. Sigal. The Quantum N-Body Problem. J. Math. Phys, 41(6) : 3348-3509, 2000.

[20] T. Kato. Fundamental Properties of Hamiltonian Operators of Schrödinger Type. Trans. Am. Math. Soc., 70 : 195-221, 1951.

[21] T. Kato. On the eigenfunctions of many particle systems in quantum mechanics. Commun. Pure and Appl. Math., 10 : 151-171, 1957.

[22] E. H. Lieb. The Stability of Matter : from Atoms to Stars. Bull. Amer. Math. Soc., 22 : 1-49, 1990.

[23] E. H. Lieb. Bound on the Maximum Negative Ionization of Atoms and Molecules. Phys. Rev. A., 29 (6) : 3018-3028, 1984.

[24] E.H. Lieb, B. Simon. The Thomas-Fermi theory of atoms, molecules and solids. Adv. Math., 23 : 22-116, 1977.

[25] E. H. Lieb, W. E. Thirring. Universal Nature of Van Der Waals Forces for Coulomb Systems. Phys. Rev. A, 34 : 40-46, 1986.

[26] P. L. Lions. The concentration-compactness principle in the calculus of variations. The locally compact case, Part I. Ann. Inst. Henri Poincaré, 1 (2) : 109-149, 1984.

[27] M. Reed, B. Simon. Methods of Modern Mathematical Physics. I - Functionnal Analysis. Academic Press, 1972.

[28] H. B. Schlegel. In Ab Inition Methods in Quantum Chemistry, Lawley Ed, 1987. Vol 1, p 249-286 and references cited therein.

[29] I.M. Sigal. Geometric methods in the quantum many-body problem. Non existence of very negative ions. Commun. Math. Phys., 85 : 309-324, 1982.

[30] B. Simon. Geometric methods in multiparticle quantum systems. Commun. Math. Phys., 55 : 259-274, 1977. 
[31] E. Teller. Rev. Mod. Phys., 34 : p 267, 1962.

[32] C. Van Winter. Theory of Finite Systems of Particles. I. The Green function. Mat.-Fys. Skr. Danske Vid. Selsk., 2 (8), 1964.

[33] G. M. Zhislin. Discussion of the spectrum of Schrödinger operators for systems of many particles. (In Russian). Trudy Moskovskogo matematiceskogo obscestva, 9 : 81-120, 1960. 